Supporting Information

\title{
Control over the Free Space within Poly(ionic liquid)s for Selective Adsorption of "Size-Matching" Dyes
}

Li Qin, ${ }^{\dagger}$ Hao Hu, ${ }^{\dagger}$ Dawei Zhang, ${ }^{*}, \dagger, \dagger$ Teng $\mathrm{Xue}^{\dagger}$ and Guohua Gao ${ }^{*, \dagger}$

${ }^{\dagger}$ Shanghai Key Laboratory of Green Chemistry and Chemical Processes, School of Chemistry and Molecular Engineering, East China Normal University, 3663 North Zhongshan Road, Shanghai.

${ }^{\ddagger}$ Department of Chemistry, University of Cambridge, Lensfield Road, Cambridge, CB2 1EW, United Kingdom.

*Prof. Guohua Gao. E-mail: ghgao@chem.ecnu.edu.cn.

*Dr. Dawei Zhang. E-mail: dz302@cam.ac.uk. 
The amount of dyes adsorbed on the PIL at any time $q_{t}(\mathrm{mg} / \mathrm{g})$ could be described as :

$$
q_{t}=\frac{C_{o}-C_{t}}{m} V
$$

where $C_{0}(\mathrm{mg} / \mathrm{L})$ is the initial concentration of dyes in solution, $C_{t}(\mathrm{mg} / \mathrm{L})$ is the dyes concentration at time $t, m(\mathrm{~g})$ is the mass of adsorbent, and $V$ is the volume of solution (L).

The pseudo-first-order and pseudo-second-order models were given as Eqs. (2) and (3), respectively,

$$
\begin{gathered}
\log \left(q_{e}-q_{t}\right)=\log q_{e}-k_{1} t \\
\frac{t}{q_{t}}=\frac{1}{k_{2} q_{e}^{2}}+\frac{t}{q_{e}}
\end{gathered}
$$

where $q_{e}(\mathrm{mg} / \mathrm{g})$ and $q_{t}(\mathrm{mg} / \mathrm{g})$ are the amounts of dyes adsorbed at equilibrium and at time $t(\mathrm{~h})$, respectively. $k_{1}(/ \mathrm{h})$ and $k_{2}(\mathrm{~g} / \mathrm{mg} \mathrm{h})$ represent the rate constant of pseudofirst-order and pseudo-second-order adsorption. And $t(\mathrm{~h})$ is the adsorption time.

The Langmuir isotherm can be expressed as:

$$
\frac{C_{e}}{q_{e}}=\frac{q}{q_{m} K_{L}}+\frac{C_{e}}{q_{m}}
$$

where $C_{e}(\mathrm{mg} / \mathrm{L})$ is the equilibrium dye concentration, $q_{e}(\mathrm{mg} / \mathrm{g})$ is the adsorption capacity at equilibrium, $q_{m}(\mathrm{mg} / \mathrm{g})$ is the maximum theoretical adsorption capacity, and $K_{L}(\mathrm{~L} / \mathrm{mg})$ is the adsorption equilibrium constant. The constants $q_{m}$ and $K_{L}$ can be calculated from the intercepts and the slopes of a linear plots of $C_{e} / q_{e}$ versus $C_{e}$. 


\section{The ${ }^{13}$ C MAS NMR spectra of poly(ionic liquid)s}
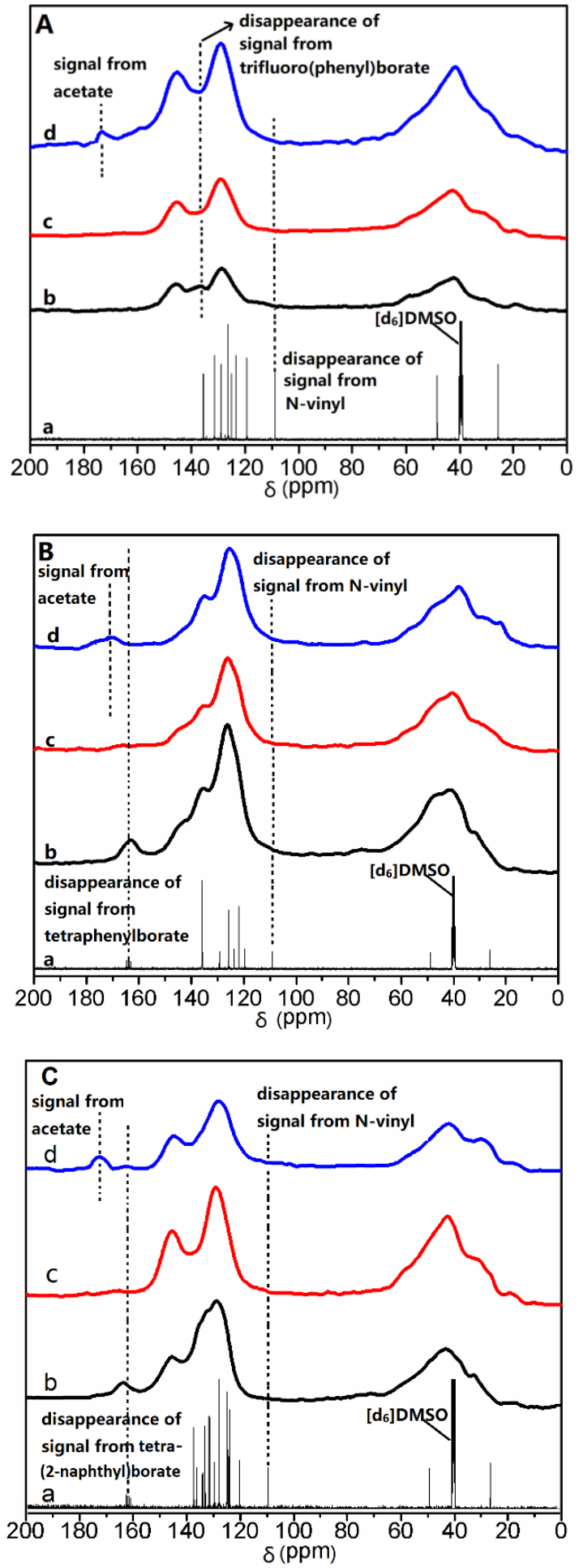

Figure S1. A: ${ }^{13} \mathrm{C}$ NMR spectrum of (a) BVImBPhF 3 in [d6]DMSO, ${ }^{13} \mathrm{C}$ MAS NMR spectra of (b) Poly(BVIm-DVB-BPhF 3 ), (c) Poly(BVIm-DVB-BPhF 3 )-Cl, (d) Poly(BVIm-DVB-BPhF 3 )-OAc;

$\mathrm{B}:{ }^{13} \mathrm{C}$ NMR spectrum of (a) $\mathrm{BVImBPh}_{4}$ in [d6]DMSO, ${ }^{13} \mathrm{C}$ MAS NMR spectra of (b)

Poly(BVIm-DVB- $\mathrm{BPh}_{4}$ ), (c) Poly(BVIm-DVB-BPh 4 )-Cl, (d) Poly(BVIm-DVB- $\mathrm{BPh}_{4}$ )-OAc; C:

${ }^{13} \mathrm{C}$ NMR spectrum of (a) BVImBNap 4 in [d6]DMSO, ${ }^{13} \mathrm{C}$ MAS NMR spectra of (b) Poly(BVIm-

DVB-BNap 4 ), (c) Poly(BVIm-DVB-BNap 4 )-Cl, (d) Poly(BVIm-DVB-BNap 4 )-OAc. 
The ${ }^{11}$ B MAS NMR spectra of poly(ionic liquid)s
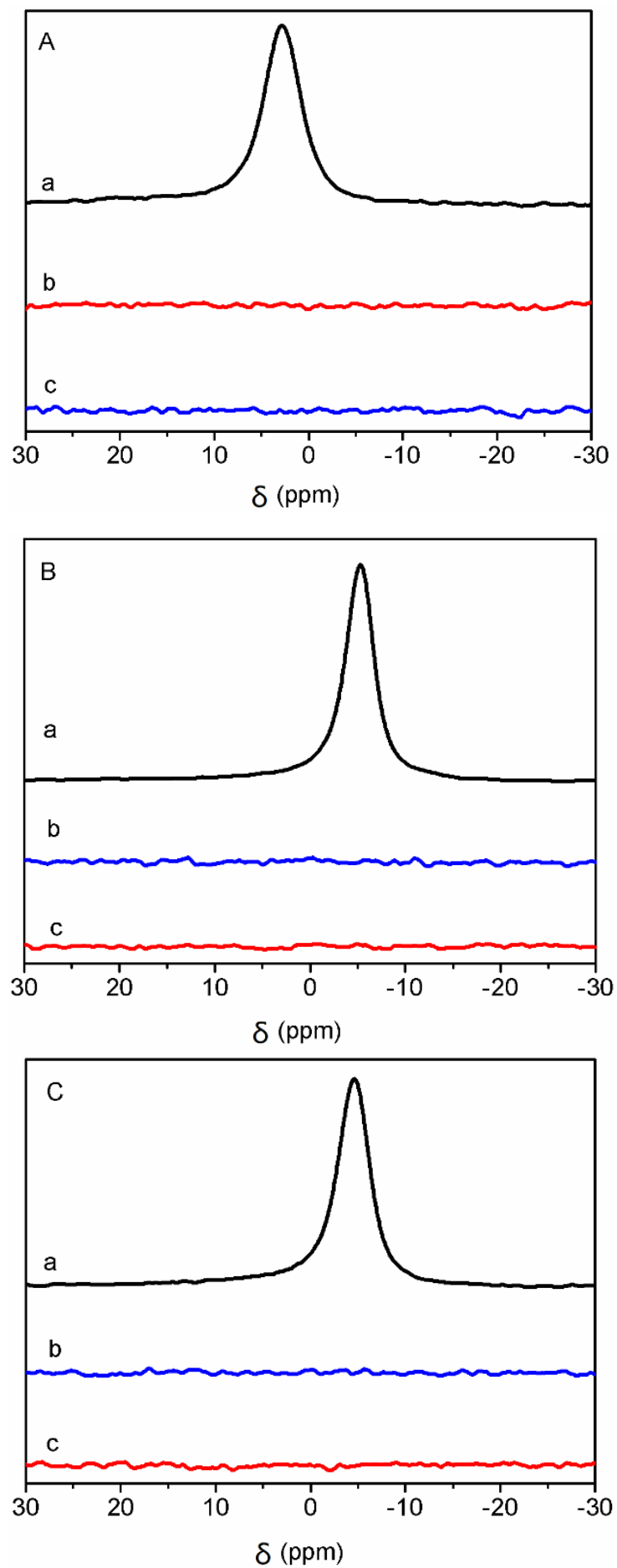

Figure S2. A: ${ }^{11}$ B MAS NMR spectra of a) Poly(BVIm-DVB-BPhF 3 ), b) Poly(BVIm-DVB$\mathrm{BPhF}_{3}$ )-Cl, c) Poly(BVIm-DVB-BPhF 3 )-OAc; $\mathrm{B}:{ }^{11} \mathrm{~B}$ MAS NMR spectra of a) Poly(BVIm-DVB$\left.\mathrm{BPh}_{4}\right)$, b) Poly(BVIm-DVB-BPh 4 )-Cl, c) Poly(BVIm-DVB-BPh $)$-OAc; C: ${ }^{11} \mathrm{~B}$ MAS NMR spectra of a) Poly(BVIm-DVB-BNap 4 ), b) Poly(BVIm-DVB-BNap 4 )-Cl, c) Poly(BVIm-DVBBNap $\left._{4}\right)-\mathrm{OAc}$. 


\section{Fourier transform infrared spectrometer}
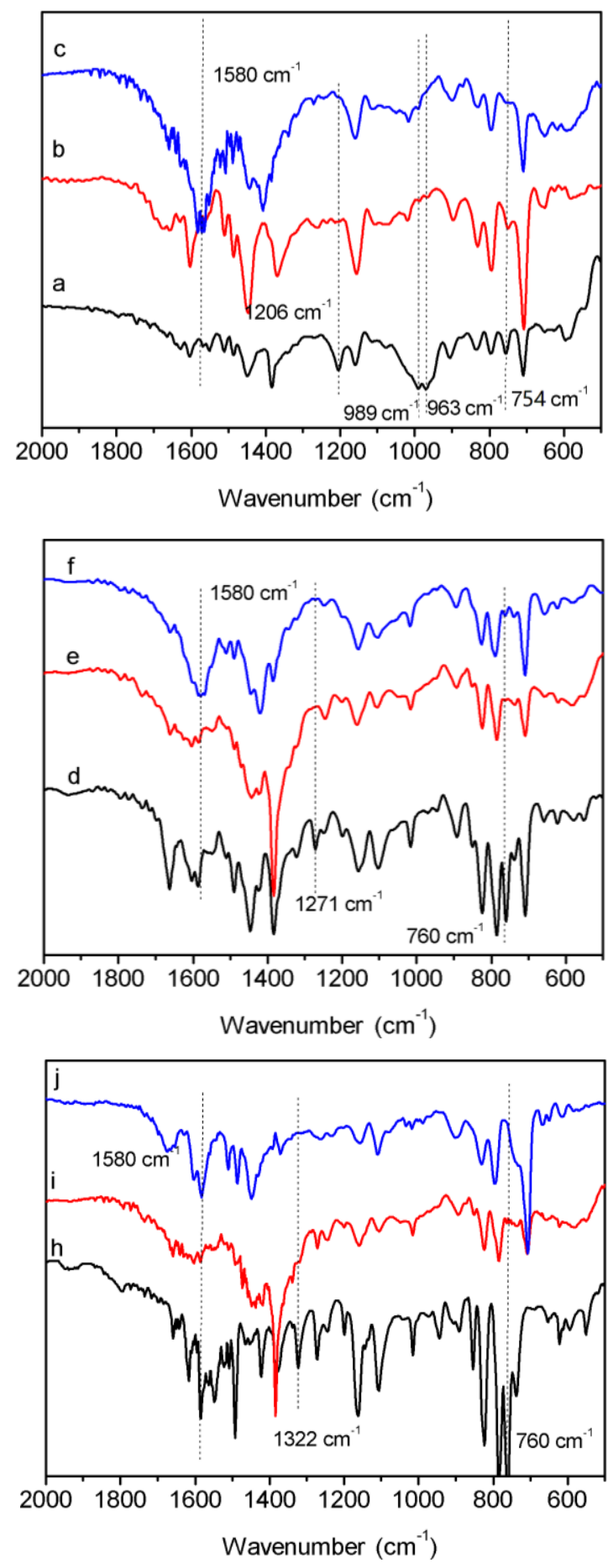

Figure S3. FT-IR spectra of (a) Poly(BVIm-DVB-BPhF ${ }_{3}$, (b) Poly(BVIm-DVB-BPhF 3 )-Cl, (c) Poly(BVIm-DVB-BPhF 3 )-OAc; (d) Poly(BVIm-DVB- BPh 4 ), (e) Poly(BVIm-DVB-BPh 4 )-Cl, (f) Poly(BVIm-DVB- BPh 4 )-OAc; (g) Poly(BVIm-DVB-BNap 4 ), (h) Poly(BVIm-DVB-BNap 4 )-Cl, (i) Poly(BVIm-DVB-BNap4)-OAc. 
Thermogravimetric analysis
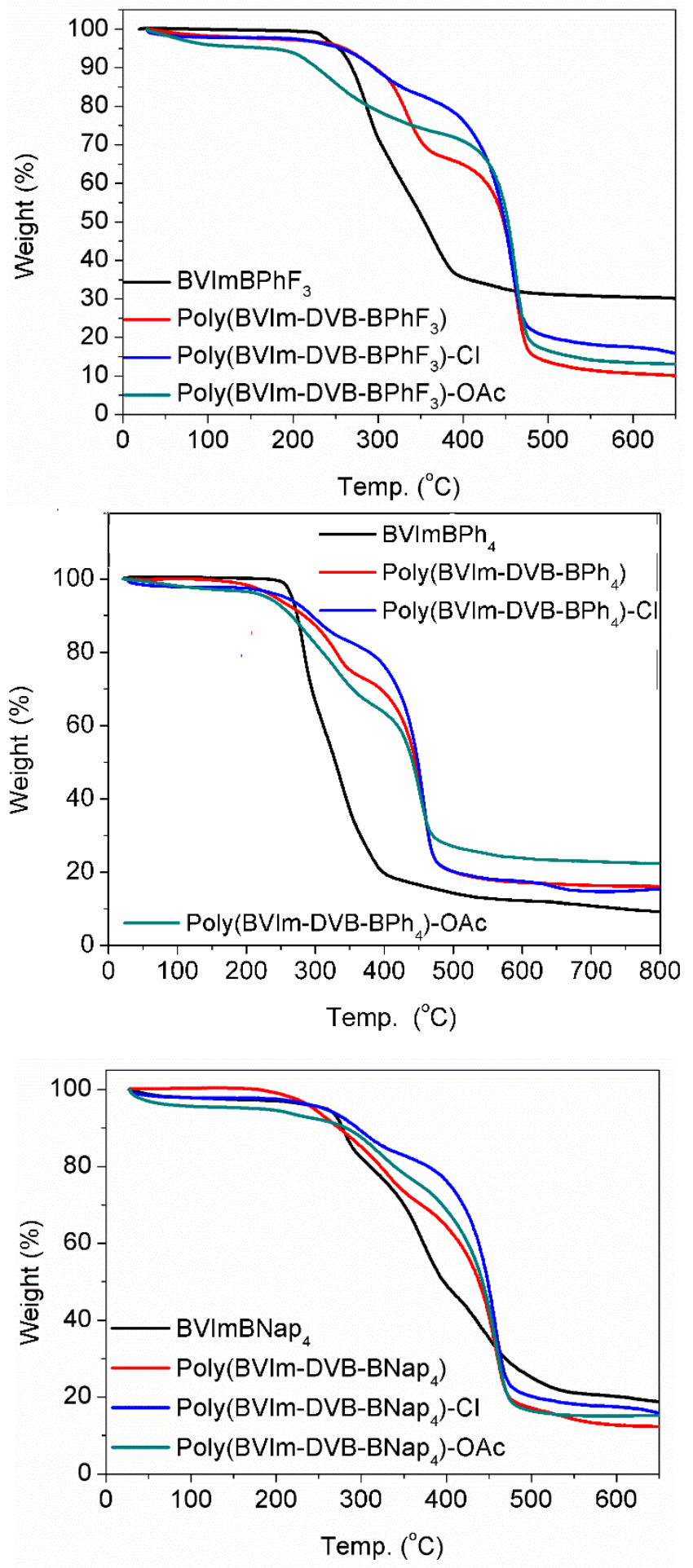

Figure S4. TGA of PILs with different anions. 


\section{Element analysis}

Table S1. Element analysis and the content of imidazolium of PILs

\begin{tabular}{|c|c|c|c|c|c|}
\hline Entry & PILs & $\mathrm{C}(\%)$ & $\mathrm{N}(\%)$ & $\mathrm{H}(\%)$ & $\operatorname{Im}(\mathrm{mmol} / \mathrm{g})$ \\
\hline 1 & Poly(BVIm-DVB-BPhF 3 ) & 70.64 & 5.02 & 8.01 & 1.79 \\
\hline 2 & Poly(BVIm-DVB-BPhF $)-O A c$ & 74.15 & 5.78 & 8.31 & 2.06 \\
\hline 3 & Poly(BVIm-DVB-BPhF 3$)-\mathrm{Cl}$ & 73.31 & 5.89 & 7.85 & 2.10 \\
\hline 4 & Poly(BVIm-DVB-BPh $\left.{ }_{4}\right)$ & 77.25 & 3.76 & 7.34 & 1.34 \\
\hline 5 & Poly(BVIm-DVB-BPh $)$-OAc & 76.34 & 5.81 & 7.93 & 2.08 \\
\hline 6 & Poly(BVIm-DVB-BPh $\left.{ }_{4}\right)-\mathrm{Cl}$ & 73.56 & 6.11 & 8.13 & 2.18 \\
\hline 7 & Poly(BVIm-DVB-BPh 4$)$ & 80.69 & 2.97 & 6.65 & 1.06 \\
\hline 8 & Poly(BVIm-DVB-BPh $\left.{ }_{4}\right)-O A c$ & 77.43 & 5.52 & 7.91 & 1.97 \\
\hline 9 & Poly(BVIm-DVB-BPh ${ }_{4}$-Cl & 73.12 & 5.90 & 7.89 & 2.11 \\
\hline 10 & Poly(BVIm-DVB-Cl) & 75.12 & 6.08 & 7.68 & 2.17 \\
\hline
\end{tabular}

\section{Scanning electron microscopy of PILs}

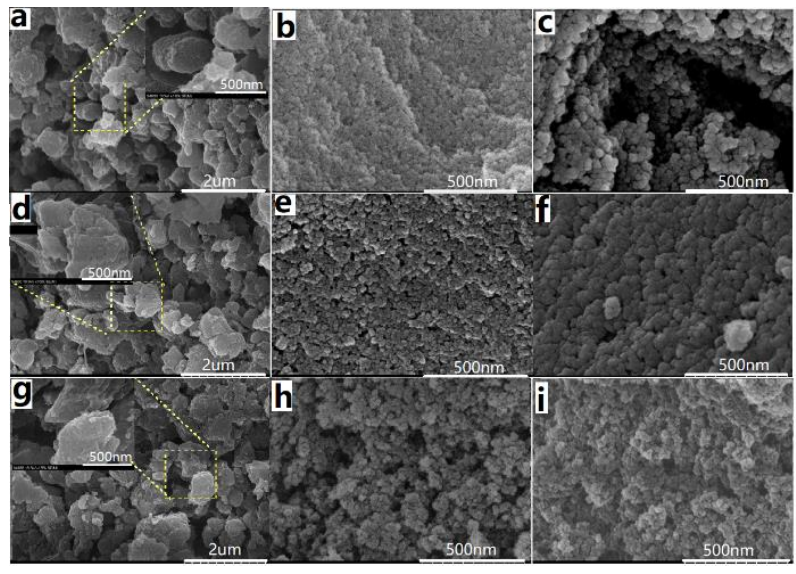

Figure S5. SEM images of PILs: (a) Poly(BVIm-DVB-BPhF 3 ), (b) Poly(BVIm-DVB-BPhF $F_{3}$-Cl,

(c) Poly(BVIm-DVB-BPhF ${ }_{3}$ )-OAc, (d) Poly(BVIm-DVB-BPh ${ }_{4}$ ), (e) Poly(BVIm-DVB-BPh $)-C l$, (f) Poly(BVIm-DVB-BPh4)-OAc, (g) Poly(BVIm-DVB-BNap4), (h) Poly(BVIm-DVB-BNap 4 )-Cl, (i) Poly(BVIm-DVB-BNap4)-OAc. 
Particle size statistic
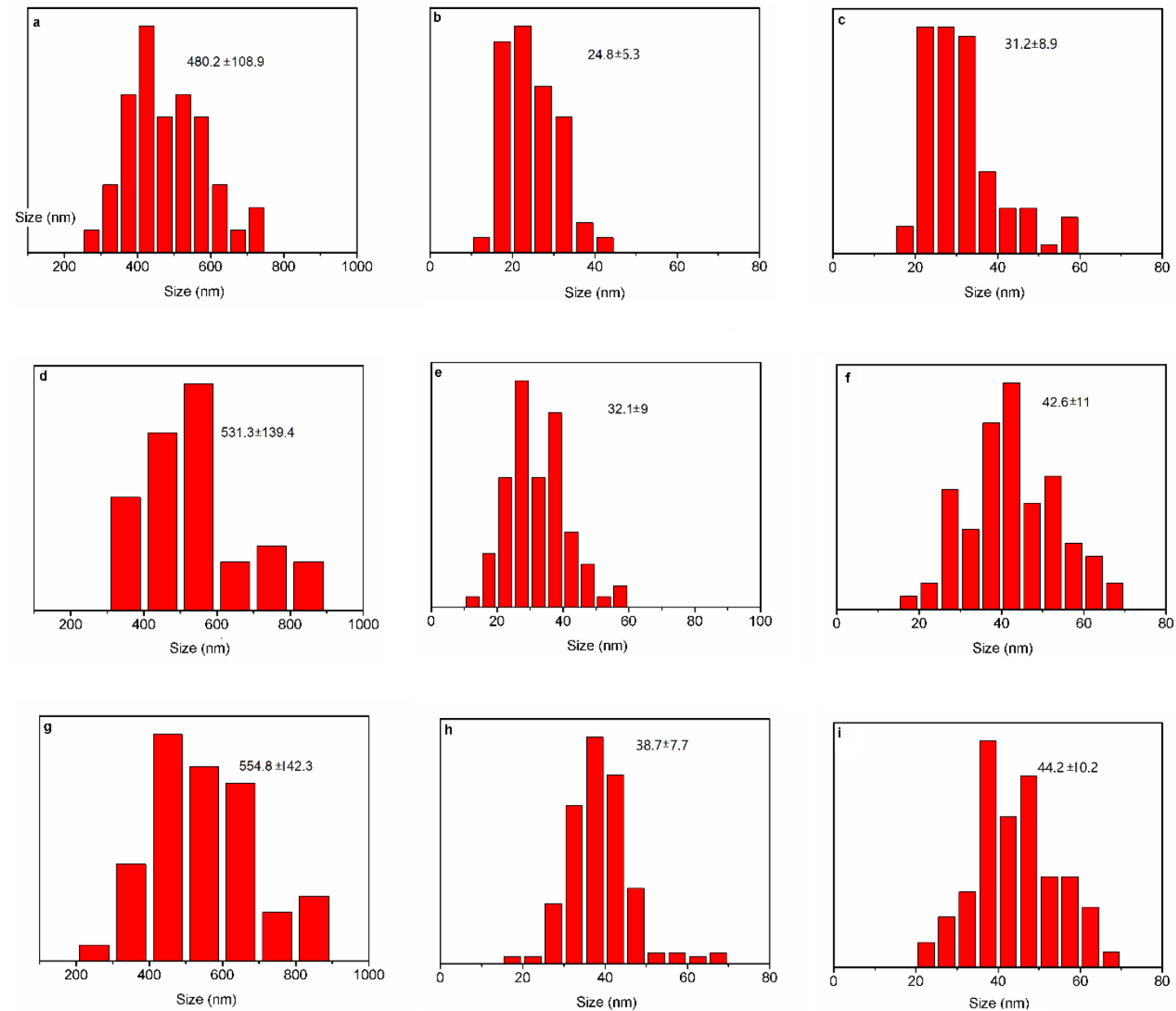

Figure S6. Particle size statistics of PILs : (a) Poly(BVIm-DVB-BPhF , $_{3}$, (b) Poly(BVIm-DVB$\mathrm{BPhF}_{3}$ )-Cl, (c) Poly(BVIm-DVB-BPhF 3 )-OAc, (d) Poly(BVIm-DVB-BPh4), (e) Poly(BVImDVB-BPh ${ }_{4}$-Cl, (f) Poly(BVIm-DVB-BPh 4 )-OAc, (g) Poly(BVIm-DVB-BNap 4 ), (h) Poly(BVImDVB-BNap4)-Cl, (i) Poly(BVIm-DVB-BNap4)-OAc. 


\section{Ar/ $\mathbf{N}_{2}$ adsorption-desorption}

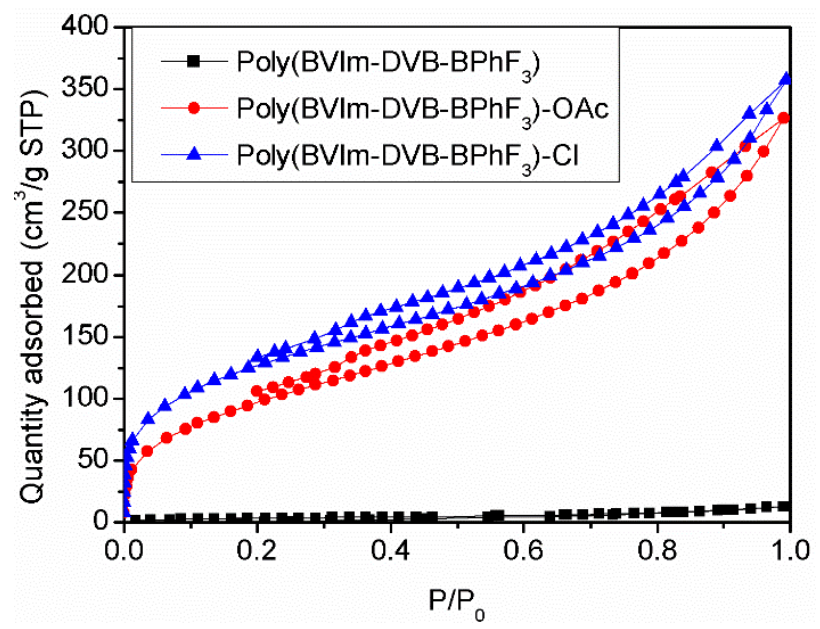

Figure S7. Ar adsorption-desorption isotherms of PILs
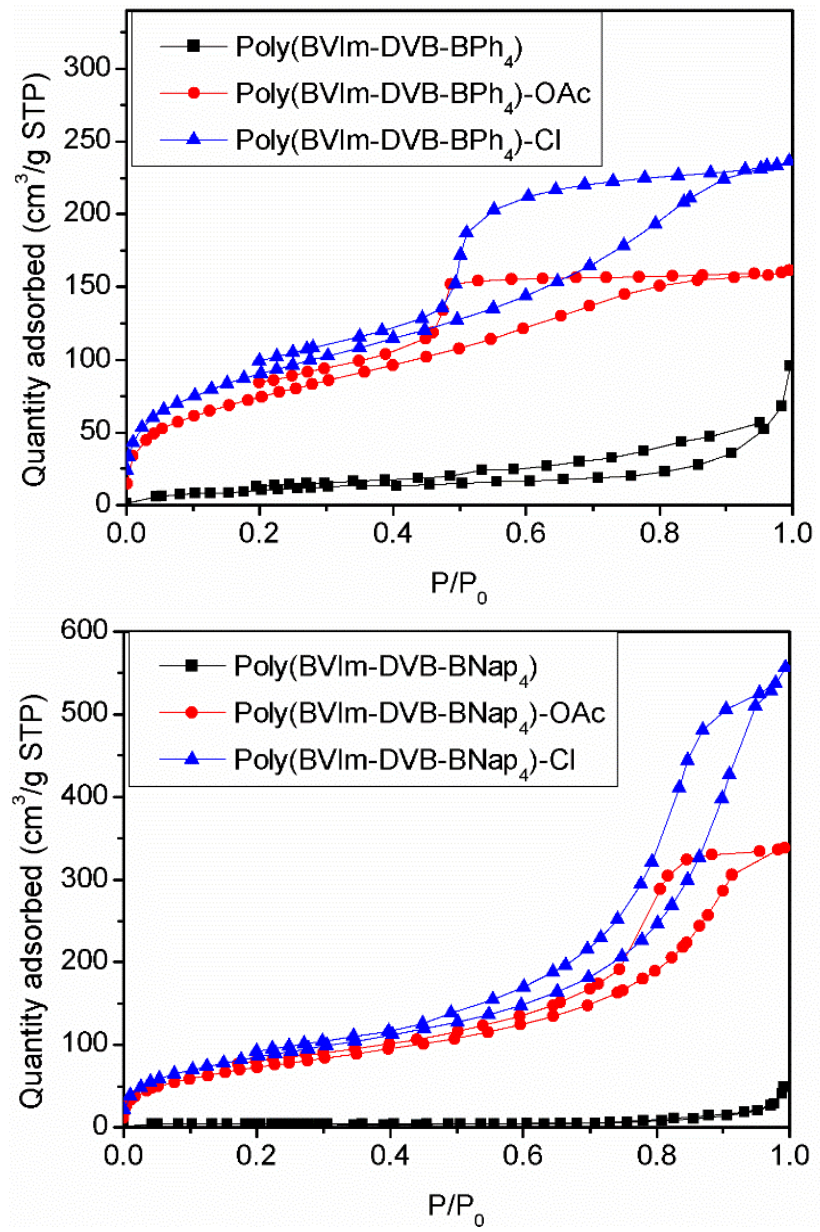

Figure S8. $\mathrm{N}_{2}$ adsorption-desorption isotherms of PILs 
Scheme S1. Synthesis of PILs with rigid structure.
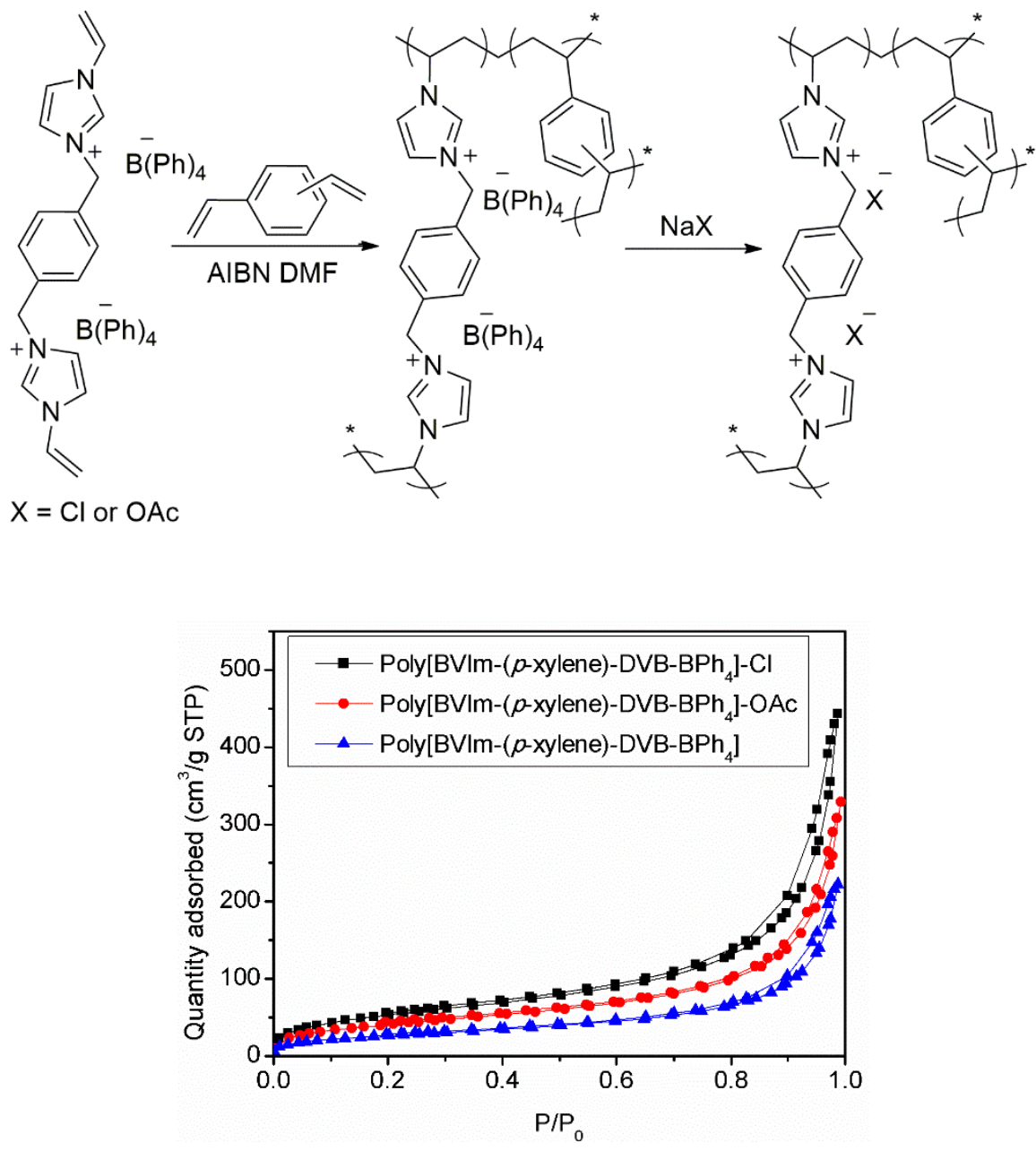

Figure S9. $\mathrm{N}_{2}$ adsorption-desorption isotherms of PILs with rigid structure.

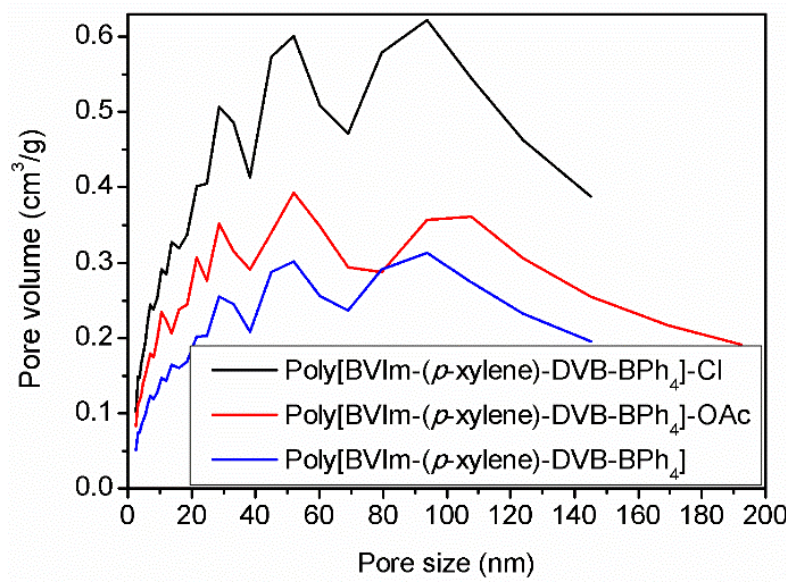

Figure S10. Pore size distribution of PILs with rigid structure. 
Table S2. The specific surface areas of the PILs prepared

\begin{tabular}{lll}
\hline Entry & PILs & $\mathrm{S}_{\text {BET }}\left(\mathrm{m}^{2} / \mathrm{g}\right)$ \\
\hline 1 & Poly[BVIm-( $p$-xylene)-DVB-BPh 4$]$ & 98.7 \\
2 & Poly[BVIm-( $p$-xylene)-DVB-BPh 4 -Cl & 196 \\
3 & Poly[BVIm-( $p$-xylene)-DVB-BPh 4 -OAc & 152 \\
\hline
\end{tabular}

The parent Poly[BVIm-(p-xylene)-DVB-BPh $\left.{ }_{4}\right]$ exhibited a specific surface area of $98.6 \mathrm{~m}^{2} / \mathrm{g}$ which was attributed to the rigid structure. After anion exchange, the increase in specific surface areas of Poly[BVIm-( $p$-xylene)-DVB-BPh 4 -Cl $\left(196 \mathrm{~m}^{2} / \mathrm{g}\right)$ and Poly[BVIm-( $p$-xylene)-DVB$\left.\mathrm{BPh}_{4}\right]$-OAc $\left(152 \mathrm{~m}^{2} / \mathrm{g}\right.$.) were not as significant as the changes of the original carbon chains-based PILs. The pore size distributions of Poly[BVIm-( $p$-xylene)-DVB-BPh $]-\mathrm{Cl} / \mathrm{OAc}$ were wider than those of the poly(ionic liquid)s with flexible carbon chains. The different phenomena may be attributed to the more rigid structures of the former that tend to support large pores, resulting in a weak effect of anion exchange on the construction of pores.
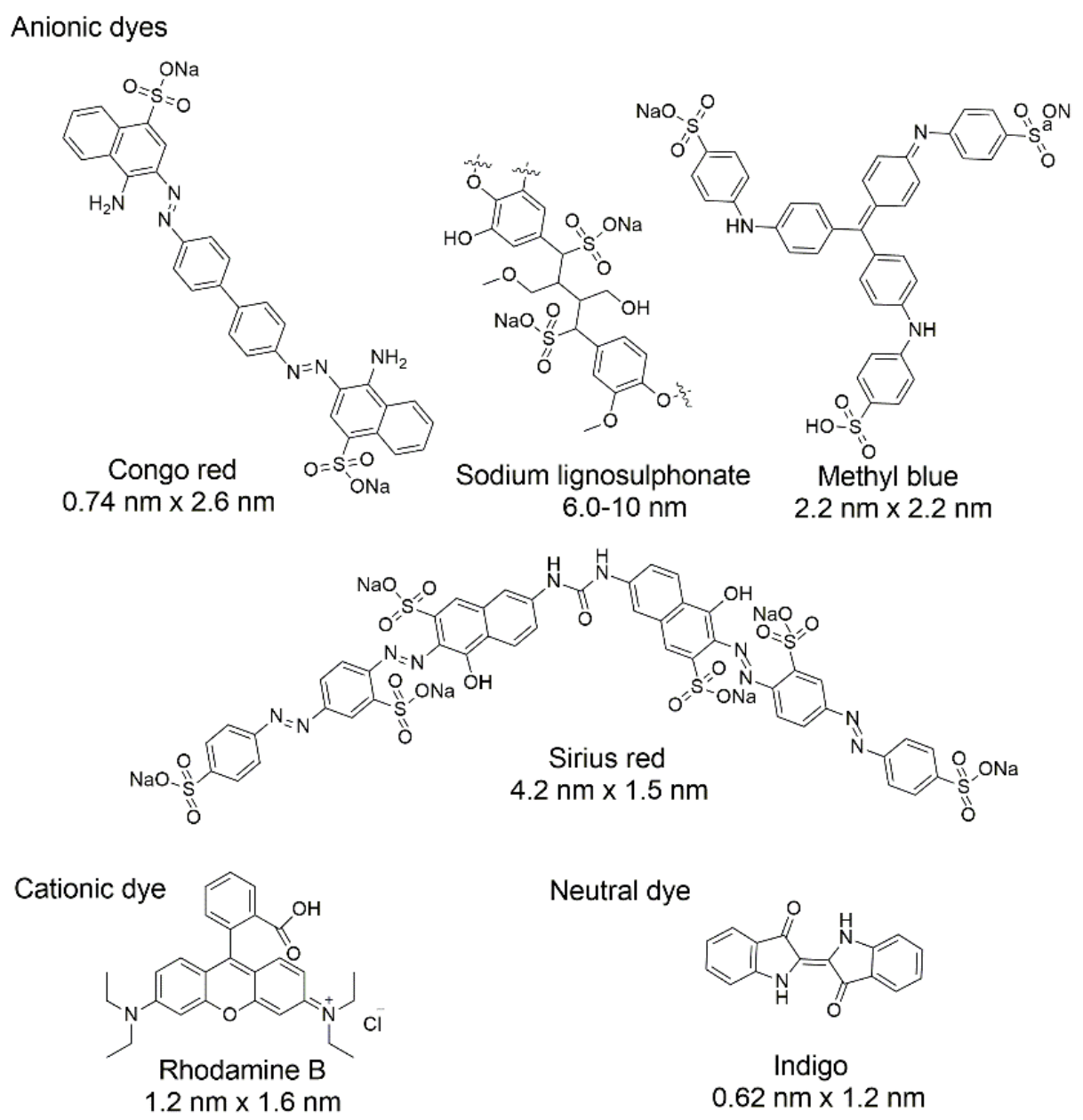

Neutral dye

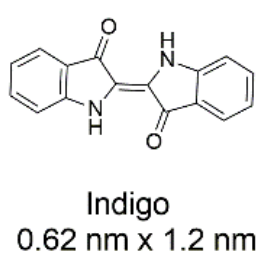

Figure S11. Molecular structures of the dyes investigated. 
Table S3. The relationship of pore size and adsorption capacity

\begin{tabular}{|c|c|c|c|c|c|c|c|}
\hline \multirow[t]{2}{*}{ Entry } & \multirow[t]{2}{*}{ PILs } & \multirow{2}{*}{$\begin{array}{l}\mathrm{S}_{\mathrm{BET}} \\
\left(\mathrm{m}^{2} / \mathrm{g}\right)\end{array}$} & \multirow{2}{*}{$\begin{array}{l}D_{\max }{ }^{a} \\
(\mathrm{~nm})\end{array}$} & \multicolumn{4}{|c|}{ Adsorption $^{\mathrm{b}}(\mathrm{mg} / \mathrm{g})$} \\
\hline & & & & $\begin{array}{l}\mathrm{CR} \\
(0.74 \mathrm{~nm} \\
\times 2.6 \mathrm{~nm})^{\mathrm{c}}\end{array}$ & $\begin{array}{l}\mathrm{SL} \\
(6.0-10 \mathrm{~nm})^{\mathrm{c}}\end{array}$ & $\begin{array}{l}\text { MB } \\
(2.2 \mathrm{~nm} \\
\times 2.2 \mathrm{~nm})^{\mathrm{c}}\end{array}$ & $\begin{array}{l}\mathrm{SR} \\
(4.2 \mathrm{~nm} \\
\times 1.5 \mathrm{~nm})^{\mathrm{c}}\end{array}$ \\
\hline 1 & $\begin{array}{c}\text { Poly(BVIm- } \\
\text { DVB- } \\
\left.\mathrm{BPhF}_{3}\right)-\mathrm{Cl}\end{array}$ & 409.4 & 0.81 & $3698.2 \pm 54.2$ & $637.6 \pm 16.0$ & $872.4 \pm 71.0$ & $847.8 \pm 14.6$ \\
\hline 2 & $\begin{array}{c}\text { Poly(BVIm- } \\
\text { DVB- } \\
\left.\mathrm{BPhF}_{3}\right)- \\
\mathrm{OAc}^{-}\end{array}$ & 309.9 & 0.78 & $2652.2 \pm 42.0$ & $616.6 \pm 6.8$ & $812.2 \pm 6.0$ & $674.2 \pm 10.8$ \\
\hline 3 & $\begin{array}{c}\text { Poly(BVIm- } \\
\text { DVB- } \\
\left.\mathrm{BPh}_{4}\right)-\mathrm{Cl}\end{array}$ & 323.3 & 8.0 & $2352.3 \pm 58.6$ & $1612.1 \pm 61.3$ & $2388.4 \pm 18.9$ & $2259.8 \pm 51.6$ \\
\hline 4 & $\begin{array}{c}\text { Poly(BVIm- } \\
\text { DVB- } \\
\left.\mathrm{BPh}_{4}\right) \text {-OAc }\end{array}$ & 270.4 & 6.0 & $2032.8 \pm 29.4$ & $\begin{array}{l}1330.0 \pm 35.4 \\
5\end{array}$ & $2198.8 \pm 11.9$ & $1989.2 \pm 23.8$ \\
\hline 5 & $\begin{array}{l}\text { Poly(BVIm- } \\
\text { DVB- } \\
\left.\text { BNap }_{4}\right)-\mathrm{Cl}\end{array}$ & 310.5 & 22 & $2172.4 \pm 34.2$ & $1053.6 \pm 47.7$ & $2351.1 \pm 44.2$ & $2056.4 \pm 51.1$ \\
\hline 6 & $\begin{array}{c}\text { Poly(BVIm- } \\
\text { DVB- } \\
\left.\text { BNap }_{4}\right)^{-} \\
\text {OAc }\end{array}$ & 260.5 & 18 & $1852.3 \pm 7.8$ & $970.9 \pm 36.3$ & $2003.8 \pm 11.6$ & $1792.4 \pm 54.1$ \\
\hline 7 & $\begin{array}{l}\text { Poly(BVIm- } \\
\text { DVB-Cl) }\end{array}$ & 23.7 & - & $632.9 \pm 18.5$ & $621.1 \pm 38.1$ & $601.4 \pm 21.6$ & $502.5 \pm 11.9$ \\
\hline
\end{tabular}

${ }^{a}$ Maximum pore diameter. ${ }^{b}$ Adsorption capacity was calculated by Langmuir isotherm. ${ }^{\mathrm{c}}$ Molecular size of dye.

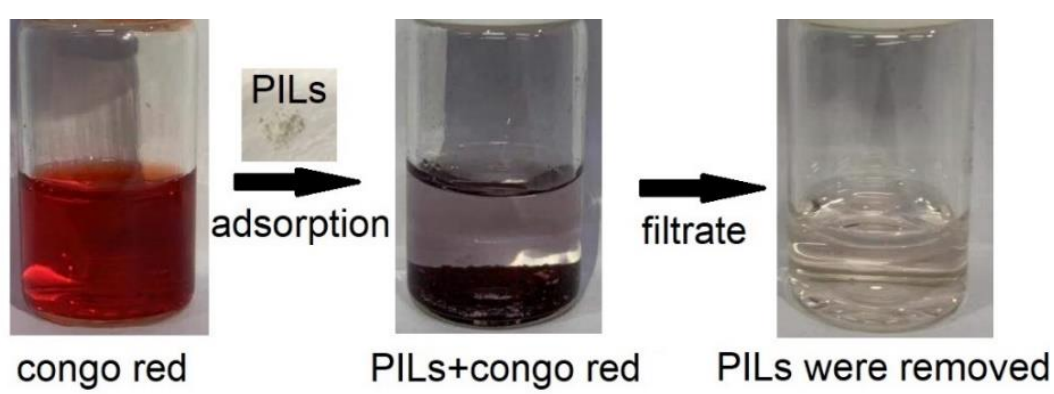

Figure S12. Images of the adsorption in congo red by Poly(BVIm-DVB-BPhF 3$)-\mathrm{Cl}$ 
Table S4. The mass change of Poly(BVIm-DVB-BPh 4$)-C l$ after dye adsorption

\begin{tabular}{llll}
\hline Entry & Dye & $\begin{array}{l}\text { Mass of PILs before } \\
\text { adsorption }(\mathrm{mg})\end{array}$ & $\begin{array}{l}\text { Mass of PILs after } \\
\text { adsorption }(\mathrm{mg})\end{array}$ \\
\hline 1 & Congo red & 5.1 & 17.2 \\
2 & Methyl blue & 5.4 & 15.4 \\
3 & Sodium lignosulphonate & 5.2 & 11.8 \\
4 & Sirius red & 5.5 & 14.8
\end{tabular}

Adsorption conditions: $25 \mathrm{~mL}, 700 \mathrm{mg} / \mathrm{L}, 25^{\circ} \mathrm{C}$, and $36 \mathrm{~h}$.

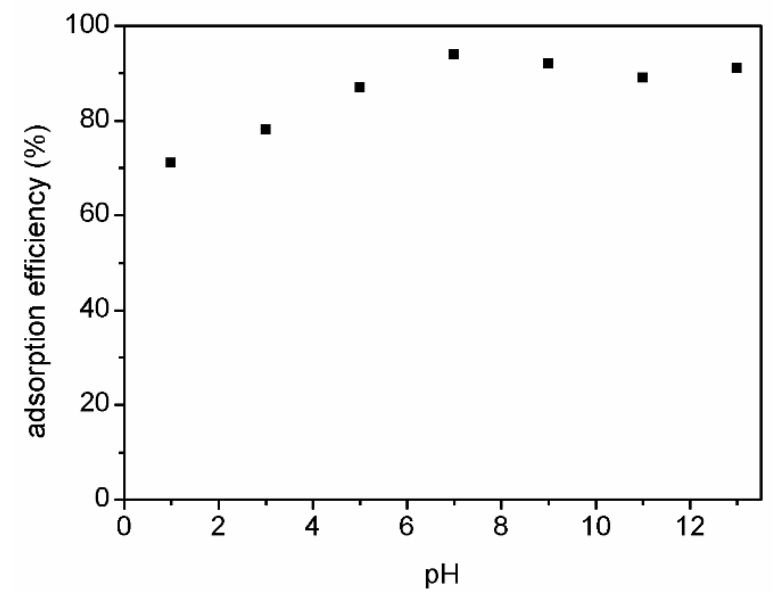

Figure S13. The adsorption efficiency of Poly(BVIm-DVB-BPh $\left.{ }_{4}\right)-\mathrm{Cl}$ on methyl blue at different pH. Conditions: $25 \mathrm{~mL}, 200 \mathrm{mg} / \mathrm{L}, 5 \mathrm{mg}$ Poly(BVIm-DVB-BPh 4$)-\mathrm{Cl}, 25^{\circ} \mathrm{C}$, and $36 \mathrm{~h}$.

Table S5. The selective adsorption capacity of PILs in dye mixture solution

\begin{tabular}{|c|c|c|c|}
\hline Entry & PILs & Mixture solution $^{\mathrm{a}}$ & Adsorption capacity (mg/g) \\
\hline \multirow{3}{*}{1} & \multirow{3}{*}{ Poly(BVIm-DVB-BPhF $)-C l$} & $\mathrm{CR} / \mathrm{MB}$ & $1000 / 135$ \\
\hline & & $\mathrm{CR} / \mathrm{SR}$ & $1000 / 105$ \\
\hline & & $\mathrm{CR} / \mathrm{SL}$ & $1000 / 70$ \\
\hline \multirow{3}{*}{$2^{b}$} & \multirow{3}{*}{ Poly(BVIm-DVB-BPh 4 )-Cl } & SL/CR & $290 / 105$ \\
\hline & & SL/MB & $265 / 145$ \\
\hline & & SL/SR & $335 / 65$ \\
\hline \multirow{2}{*}{3} & \multirow{2}{*}{ Poly(BVIm-DVB-BNap4)-Cl } & SL/SR & $320 / 440$ \\
\hline & & $\mathrm{CR} / \mathrm{MB}$ & $500 / 440$ \\
\hline
\end{tabular}

${ }^{a}$ Adsorption conditions: the initial concentration of each dyes in mixture solution $\mathrm{C}_{0}=200 \mathrm{mg} / \mathrm{L}$, temperature $25^{\circ} \mathrm{C} .{ }^{\mathrm{b}}$ the initial concentration of each dyes in mixture solution $\mathrm{C}_{0}=100 \mathrm{mg} / \mathrm{L}$ 


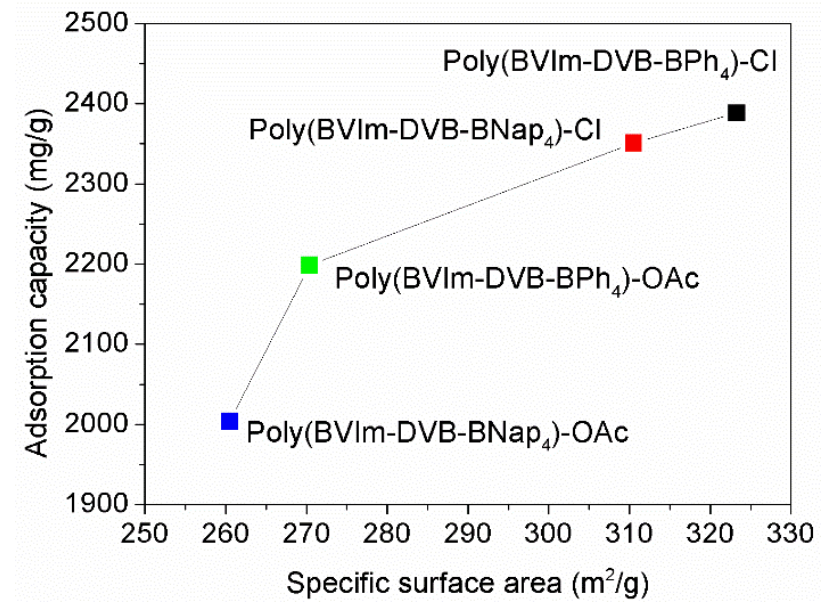

Figure S14. The relationship of adsorption capacity for methyl blue and specific surface area of PILs.

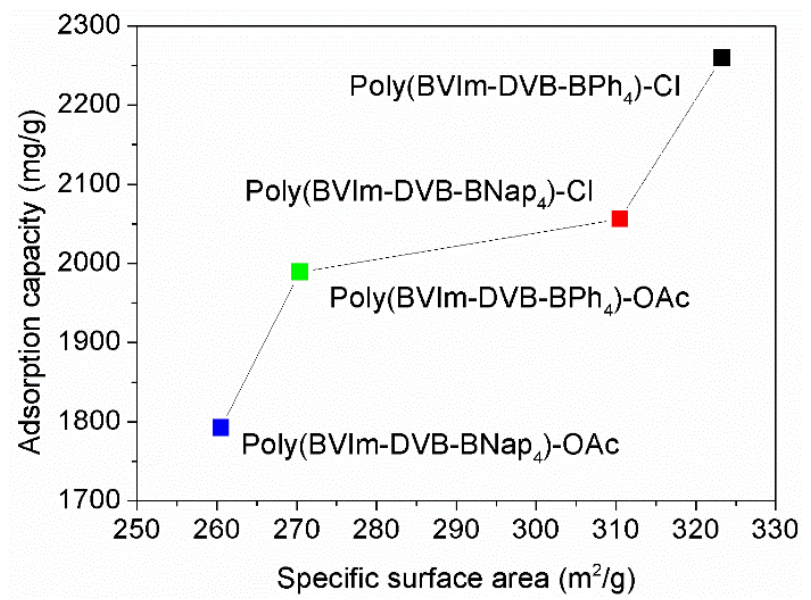

Figure S15. The relationship of adsorption capacity for sirius red and specific surface area of PILs.

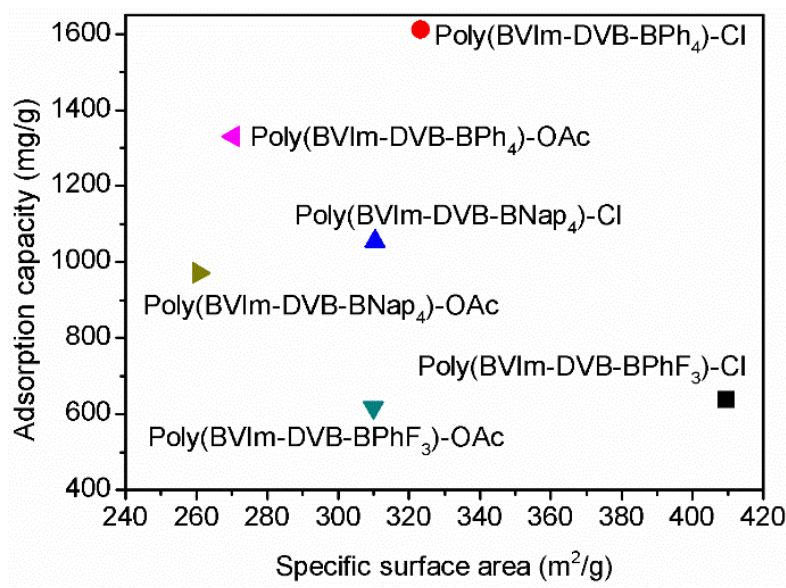

Figure S16. The relationship of adsorption capacity for sodium lignosulphonate and specific surface area of PILs. 


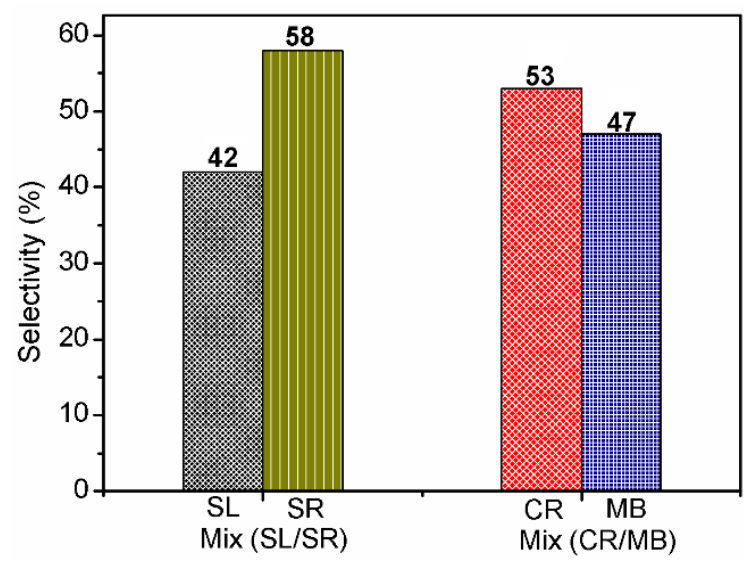

Figure S17. The selective adsorptions in dye mixtures by Poly(BVIm-DVB-BNap 4 )-Cl. Conditions: $25 \mathrm{~mL}, 200 \mathrm{mg} / \mathrm{L}$ in each dye, $5 \mathrm{mg}$ PILs, $25^{\circ} \mathrm{C}$, and $36 \mathrm{~h}$. Note: congo red (CR), methyl blue (MB), sirius red (SR) and sodium lignosulphonate (SL).

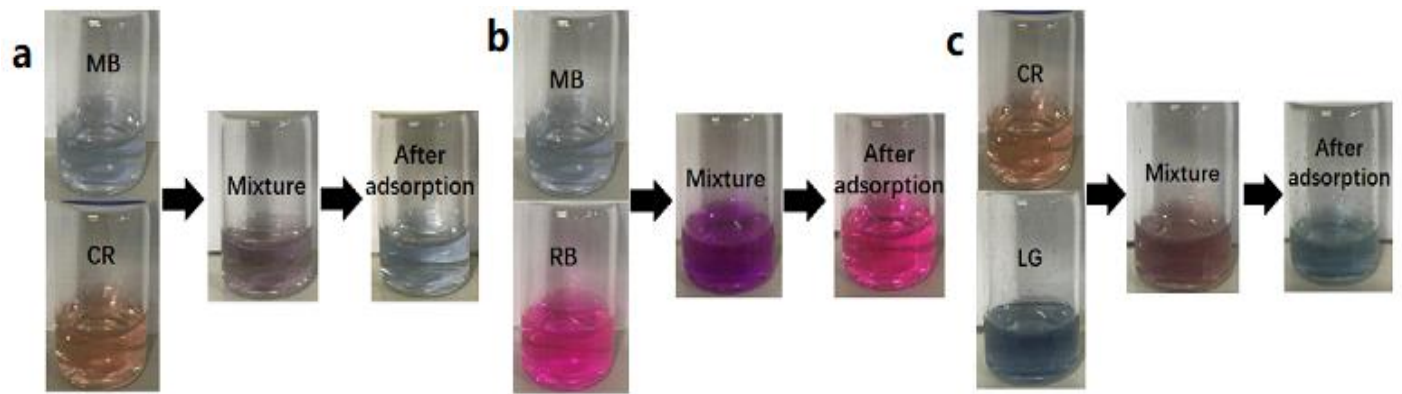

Figure S18. Images of the selective adsorption in dye mixtures by Poly(BVIm-DVB-BPhF3)-Cl (a) and Poly(BVIm-DVB-BPh4)-Cl (b and c). Adsorption conditions: the initial concentrations of all dyes $\mathrm{C} 0=200 \mathrm{ppm}$, temperature $25^{\circ} \mathrm{C}$ (the images of dye solutions were diluted by 20 times).

Table S6. The cation and non-ionic dye adsorption capacity of PILs

\begin{tabular}{|c|c|c|c|}
\hline \multirow[t]{2}{*}{ Entry } & \multirow[t]{2}{*}{ PILs } & \multicolumn{2}{|c|}{ Adsorption capacity(mg/g) } \\
\hline & & RB & IG \\
\hline 1 & Poly(BVIm-DVB-BPhF 3 )-Cl & 154.8 & 374.5 \\
\hline 2 & Poly(BVIm-DVB-BPh $\left.{ }_{4}\right)-\mathrm{Cl}$ & 235.8 & 274.7 \\
\hline 3 & Poly(BVIm-DVB-BNap 4 )-Cl & 202.4 & 240.4 \\
\hline 4 & Poly(BVIm-DVB-BPhF 3 )-OAc & 131.4 & 326.8 \\
\hline 5 & Poly(BVIm-DVB-BPh 4$)-O A c$ & 192.3 & 221.5 \\
\hline 6 & Poly(BVIm-DVB-BNap 4 )-OAc & 168.4 & 210.1 \\
\hline
\end{tabular}

${ }^{\mathrm{a}}$ Max adsorption capacity was calculated by Langmuir isotherm. 


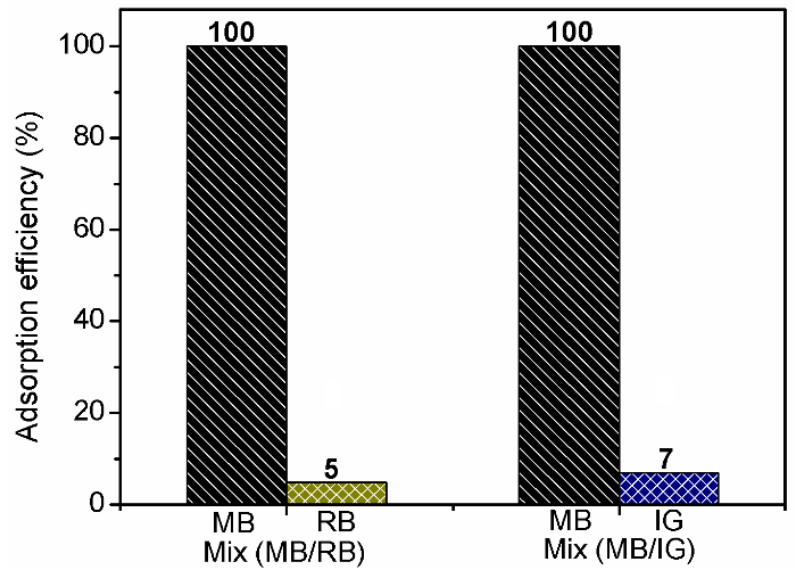

Figure S19. Selective adsorption of dyes among mixtures by Poly(BVIm-DVB-BPh 4$)-\mathrm{Cl}$.

Conditions: $25 \mathrm{~mL}, 200 \mathrm{mg} / \mathrm{L}$ in each dye, $5 \mathrm{mg}$ PILs, $25^{\circ} \mathrm{C}$ and $36 \mathrm{~h}$. Note: methyl blue (MB), rhodamine $\mathrm{B}(\mathrm{RB})$ and indigo (IG) 


\section{Adsorption kinetics of PILs}

Table S7. Adsorption pseudo second order kinetic parameters of congo red ${ }^{\mathrm{a}}$

\begin{tabular}{lllllll}
\hline Entry & Abs & Cad & Ct & qt & t(h) & t/qt \\
\hline 1 & 0.178 & 14.872 & 148.723 & 1256.383 & 0.17 & 0.000133 \\
2 & 0.163 & 13.618 & 136.179 & 1319.106 & 0.33 & 0.000253 \\
3 & 0.145 & 12.146 & 121.464 & 1392.681 & 0.50 & 0.000359 \\
4 & 0.127 & 10.601 & 106.008 & 1469.957 & 1.00 & 0.00068 \\
5 & 0.105 & 8.694 & 86.945 & 1565.277 & 1.50 & 0.000958 \\
6 & 0.093 & 7.688 & 76.877 & 1615.617 & 2.00 & 0.001238 \\
7 & 0.092 & 7.603 & 76.034 & 1619.83 & 2.50 & 0.00154 \\
8 & 0.092 & 7.564 & 75.642 & 1621.787 & 3.00 & 0.00185 \\
9 & 0.071 & 5.762 & 57.626 & 1711.872 & 4.00 & 0.00234 \\
10 & 0.034 & 2.632 & 52.647 & 1736.766 & 6.00 & 0.00346 \\
11 & 0.029 & 2.241 & 44.817 & 1740.397 & 8.00 & 0.00460 \\
\hline adye concentration $=400 \mathrm{mg} / \mathrm{L}$ & $25 \mathrm{~mL}$, Poly(BVIm-DVB-BPh $)-\mathrm{Cl}=5 \mathrm{mg}$
\end{tabular}

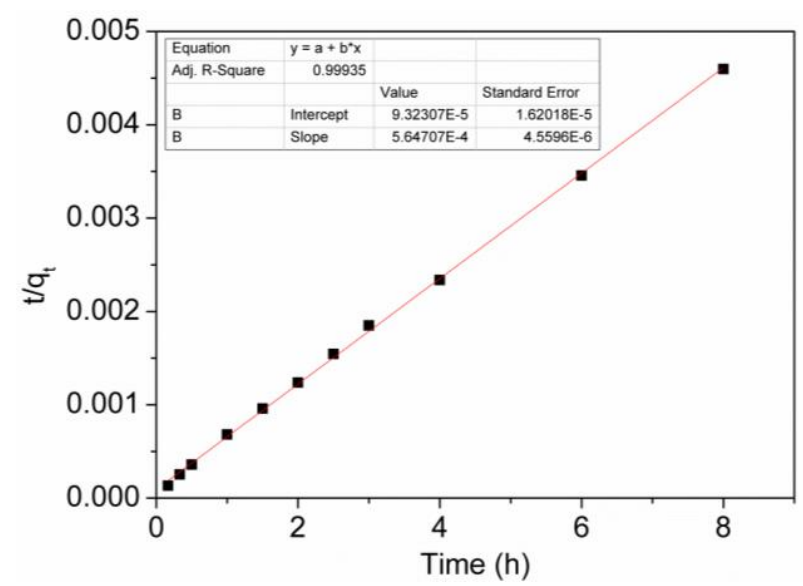

Figure S20. The adsorption pseudo second order kinetic curve of Poly(BVIm-DVB-BPh 4$)-\mathrm{Cl}$ on congo red. 
Table S8. Adsorption pseudo second order kinetic parameters of methyl blue ${ }^{\mathrm{a}}$

\begin{tabular}{ccccccc}
\hline Entry & Abs & Cad & Ct & T(h) & qt & t/qt \\
\hline 1 & 0.360 & 12.573 & 251.468 & 0.17 & 742.663 & 0.000224 \\
2 & 0.341 & 11.899 & 237.976 & 0.33 & 810.122 & 0.000411 \\
3 & 0.340 & 11.857 & 237.147 & 0.5 & 814.266 & 0.000614 \\
4 & 0.301074 & 10.508 & 210.170 & 1 & 949.152 & 0.001054 \\
5 & 0.277 & 9.669 & 193.384 & 1.5 & 1033.080 & 0.001452 \\
6 & 0.274 & 9.574 & 191.478 & 2 & 1042.613 & 0.001918 \\
7 & 0.256 & 8.948 & 178.972 & 4 & 1151.189 & 0.003475 \\
8 & 0.195 & 6.830 & 136.601 & 6 & 1316.996 & 0.004556 \\
9 & 0.184 & 6.436 & 128.730 & 8 & 1356.349 & 0.005898 \\
10 & 0.156889 & 5.502059 & 110.0412 & 10 & 1449.794 & 0.006898 \\
11 & 0.131004 & 4.603277 & 92.06553 & 12 & 1539.672 & 0.007794 \\
12 & 0.129301 & 4.544138 & 90.88275 & 24 & 1545.586 & 0.015528 \\
\hline
\end{tabular}

${ }^{\text {adye }}$ concentration $=400 \mathrm{mg} / \mathrm{L}, 25 \mathrm{~mL}$, Poly $\left(\mathrm{BVIm}-\mathrm{DVB}-\mathrm{BPh}_{4}\right)-\mathrm{Cl}=5 \mathrm{mg}$

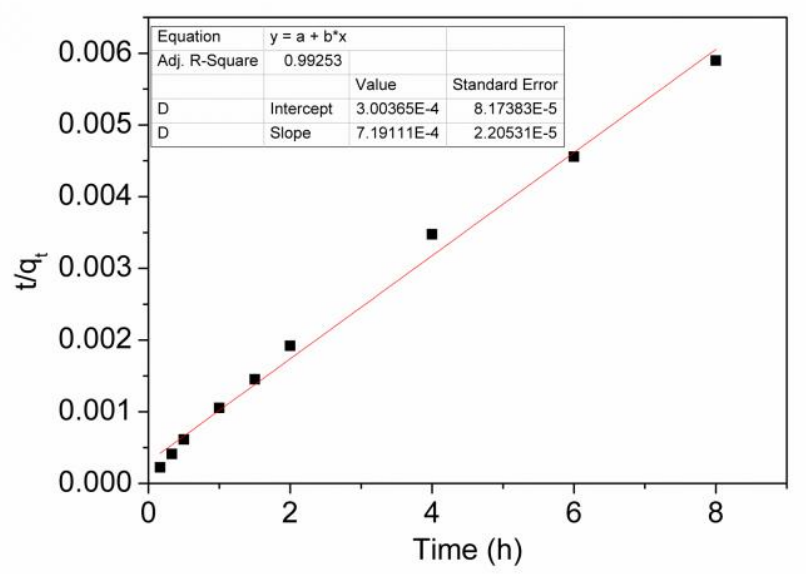

Figure S21. The adsorption pseudo second order kinetic curve of Poly(BVIm-DVB-BPh 4$)-\mathrm{Cl}$ on methyl blue. 
Table S9. Adsorption pseudo second order kinetic parameters of sirius red ${ }^{\mathrm{a}}$

\begin{tabular}{ccccccc}
\hline Entry & Abs & $\mathrm{Cad}$ & $\mathrm{Ct}$ & $\mathrm{qt}$ & $\mathrm{T}(\mathrm{h})$ & $\mathrm{t} / \mathrm{qt}$ \\
\hline 1 & 0.287 & 18.044 & 180.438 & 1097.813 & 0.50 & 0.000455 \\
2 & 0.282 & 17.720 & 177.201 & 1113.994 & 1.00 & 0.000898 \\
3 & 0.276 & 17.360 & 173.603 & 1131.983 & 1.5 & 0.001325 \\
4 & 0.275 & 17.302 & 173.025 & 1134.875 & 2 & 0.001762 \\
5 & 0.270 & 16.965 & 169.647 & 1151.765 & 2.5 & 0.002171 \\
6 & 0.252 & 15.880 & 158.801 & 1205.994 & 3 & 0.002488 \\
7 & 0.250 & 15.732 & 157.317 & 1213.415 & 3.5 & 0.002884 \\
8 & 0.239 & 15.047 & 150.468 & 1247.661 & 4 & 0.003206 \\
9 & 0.216 & 13.598 & 135.979 & 1320.105 & 6 & 0.004545 \\
10 & 0.205 & 12.896 & 128.957 & 1355.217 & 8 & 0.005903 \\
11 & 0.193 & 12.146 & 121.465 & 1392.674 & 10 & 0.00718 \\
12 & 0.168 & 10.599 & 105.990 & 1470.048 & 12 & 0.008163 \\
13 & 0.147 & 9.038 & 90.379 & 1548.107 & 14 & 0.009043 \\
\hline
\end{tabular}

adye concentration $=400 \mathrm{mg} / \mathrm{L}, 25 \mathrm{~mL}$, Poly(BVIm-DVB-BPh 4$)-C l=5 \mathrm{mg}$

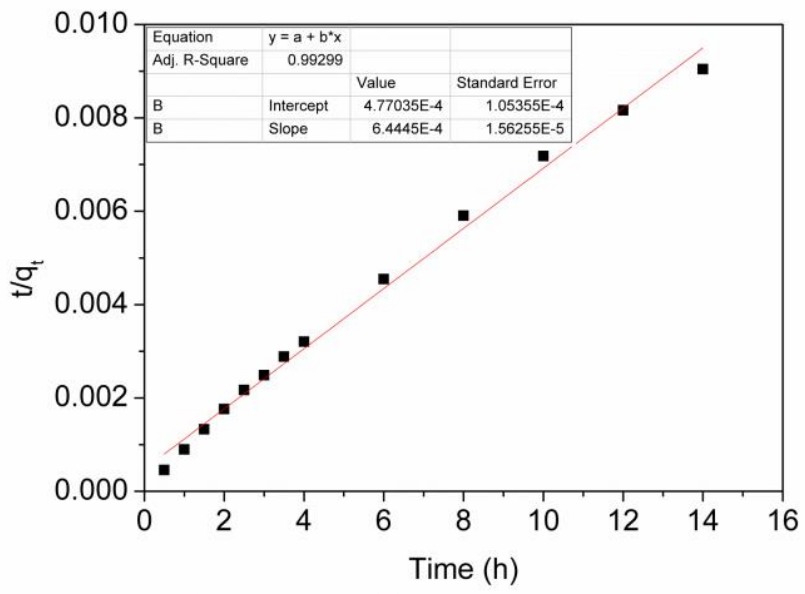

Figure S22. The adsorption pseudo second order kinetic curve of Poly(BVIm-DVB-BPh 4 -Cl on sirius red. 
Table S10. Adsorption pseudo second order kinetic parameters of sodium lignosulphonate ${ }^{\text {a }}$

\begin{tabular}{ccccccc}
\hline Entry & $\mathrm{Abs}$ & $\mathrm{Cad}$ & $\mathrm{Ct}$ & $\mathrm{qt}$ & $\mathrm{T}(\mathrm{h})$ & $\log (\mathrm{qe}-\mathrm{qt})$ \\
\hline 1 & 0.149 & 18.895 & 377.899 & 108.846 & 1 & 2.840 \\
2 & 0.278 & 35.837 & 358.368 & 200.877 & 2 & 2.778 \\
3 & 0.252 & 32.3866 & 323.856 & 359.783 & 4 & 2.645 \\
4 & 0.232 & 29.854 & 298.538 & 479.406 & 6 & 2.507 \\
5 & 0.196 & 25.108 & 251.083 & 696.188 & 12 & 2.092 \\
6 & 0.364 & 47.208 & 236.040 & 788.266 & 24 & 1.109 \\
7 & 0.178 & 22.678 & 226.781 & 801.137 & 36 & --- \\
\hline
\end{tabular}

adye concentration $=400 \mathrm{mg} / \mathrm{L}, 25 \mathrm{~mL}$, Poly(BVIm-DVB-BPh $\left.{ }_{4}\right)-\mathrm{Cl}=5 \mathrm{mg}$

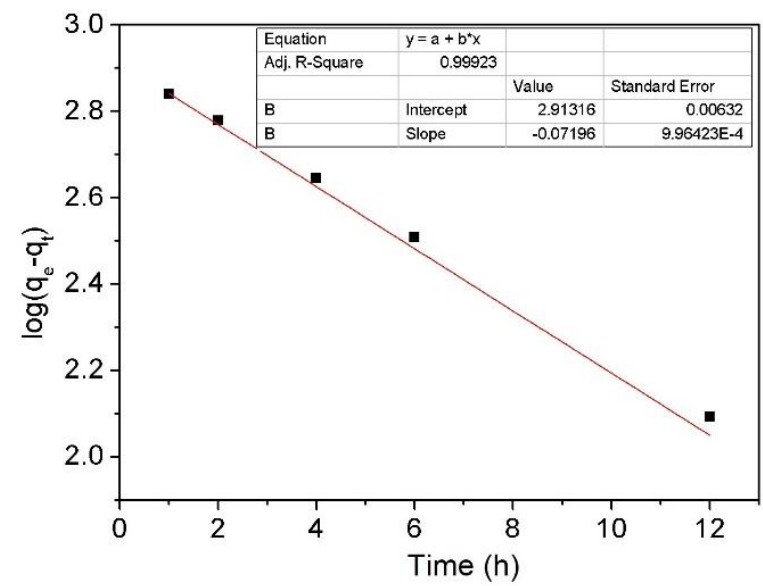

Figure S23. The adsorption pseudo first order kinetic curve of Poly(BVIm-DVB-BPh $\left.{ }_{4}\right)-\mathrm{Cl}$ on sodium lignosulphonate.

Table S11. The kinetic parameter study of PILs

\begin{tabular}{|c|c|c|c|c|}
\hline Entry & Sample & $\mathrm{R}^{2}$ & $k_{2}(\mathrm{mg} / \mathrm{gh})$ & $\mathrm{q}_{\mathrm{e}}(\mathrm{mg} / \mathrm{g})$ \\
\hline 1 & Congo red & 0.99935 & 0.003420 & 1740.4 \\
\hline 2 & Methyl blue & 0.99253 & 0.00172 & 1545.6 \\
\hline 3 & Sirius red & 0.99299 & 0.000871 & 1548.1 \\
\hline $4^{\mathrm{a}}$ & $\begin{array}{c}\text { Sodium } \\
\text { lignosulphonate }\end{array}$ & 0.99923 & 0.07196 & 818.8 \\
\hline
\end{tabular}

${ }^{\text {a }}$ Pseudo first order kinetic parameters 


\section{Recyclability of PILs}
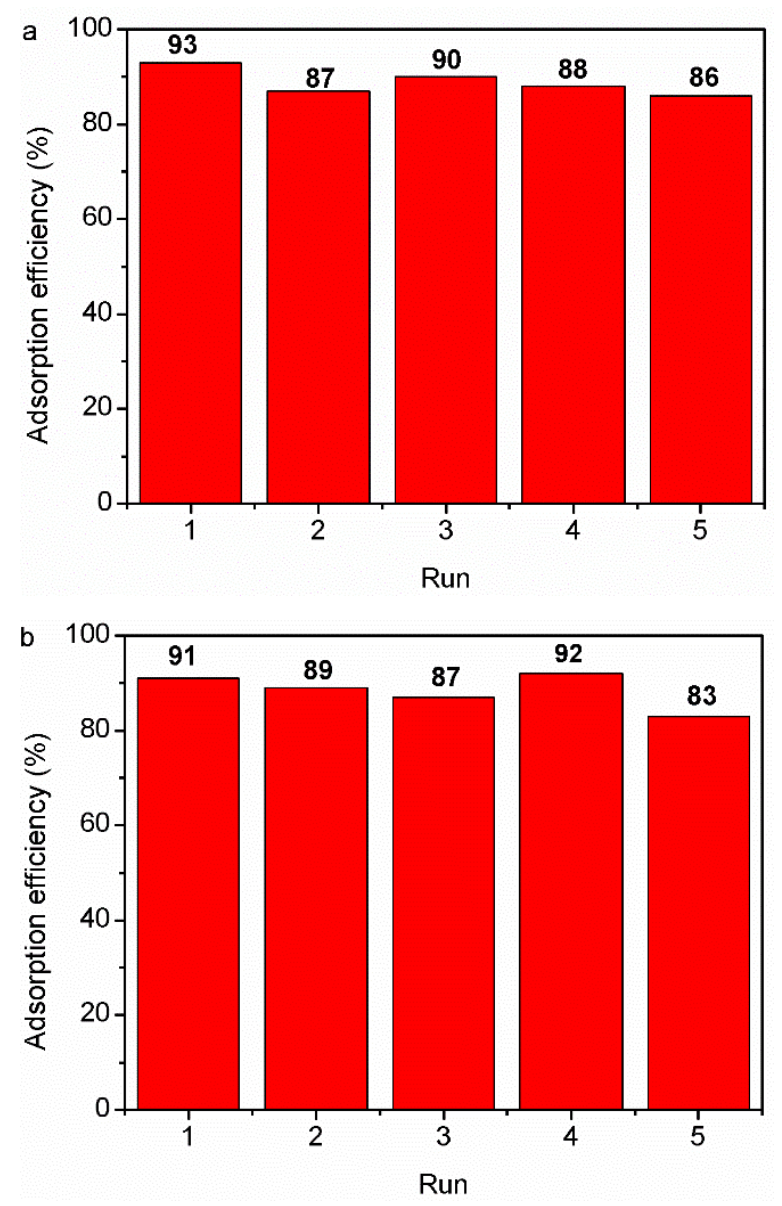

Figure S24. Recyclability of Poly(BVIm-DVB-BPh 4$)-C l$ (a) and Poly(BVIm-DVB-BPh 4 )-OAc (b) for the adsorption of methyl blue. Conditions: $25 \mathrm{~mL}, 200 \mathrm{mg} / \mathrm{L}, 5 \mathrm{mg}$ PILs, $25^{\circ} \mathrm{C}$, and $36 \mathrm{~h}$.
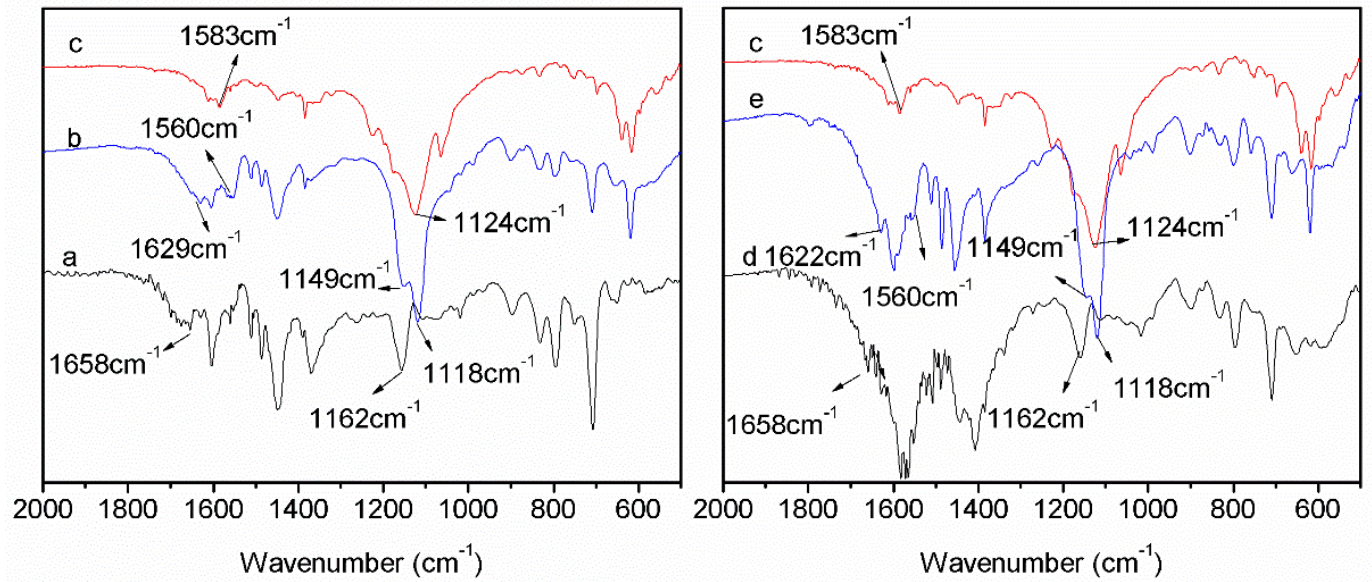

Figure S25. FT-IR spectra of (a) Poly(BVIm-DVB-BPhF 3 )-Cl, (b) Poly(BVIm-DVB-BPhF ${ }_{3}$-Cl after absorbed congo red, (c) congo red; (d) Poly(BVIm-DVB-BPhF 3 )-OAc, (e) Poly(BVIm$\left.\mathrm{DVB}-\mathrm{BPhF}_{3}\right)-\mathrm{OAc}$ after absorbed congo red. 


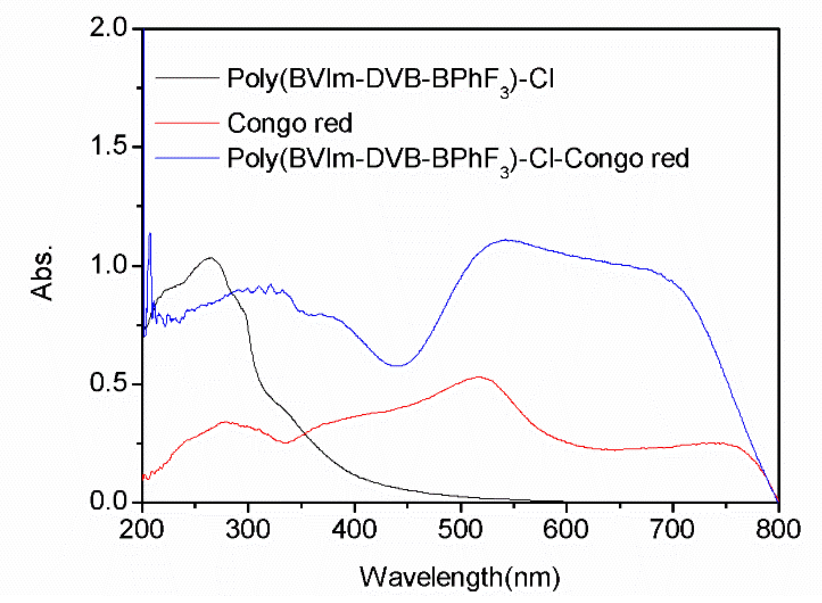

Figure S26. UV-vis spectra of Poly(BVIm-DVB-BPhF 3 -Cl, congo red and Poly(BVIm-DVB$\left.\mathrm{BPhF}_{3}\right)-\mathrm{Cl}$ after absorbed congo red.

UV-vis spectra did not provide much useful information due to the low resolution of solid samples.

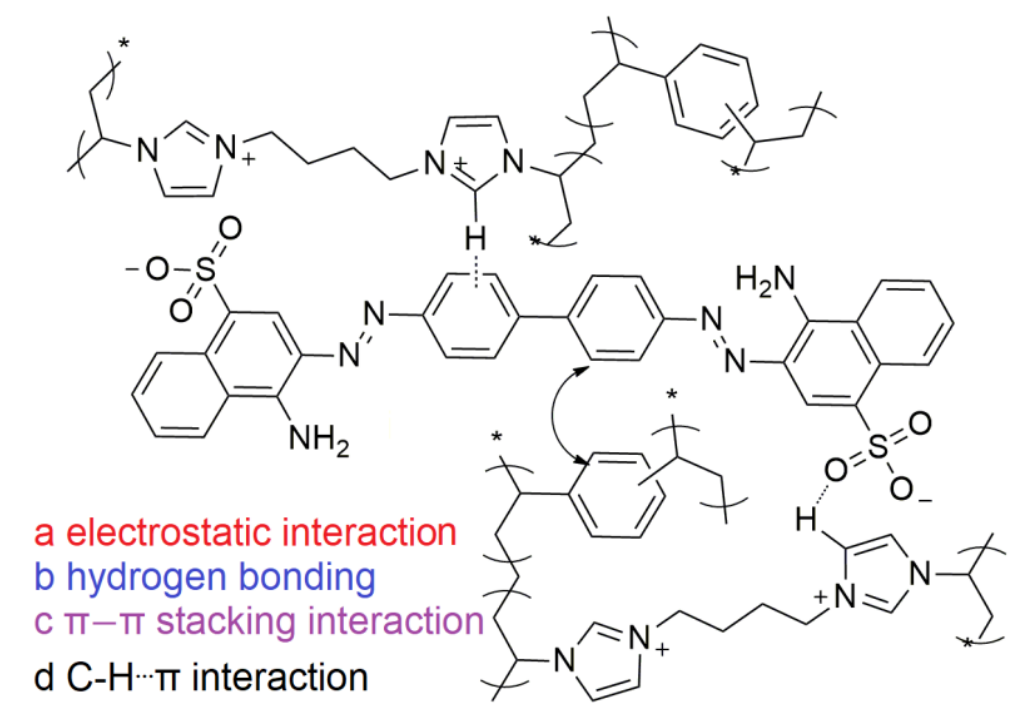

Figure S27. The possible mechanism of adsorption of PILs on dye molecules. 


\section{Dye adsorption curve of PILs}

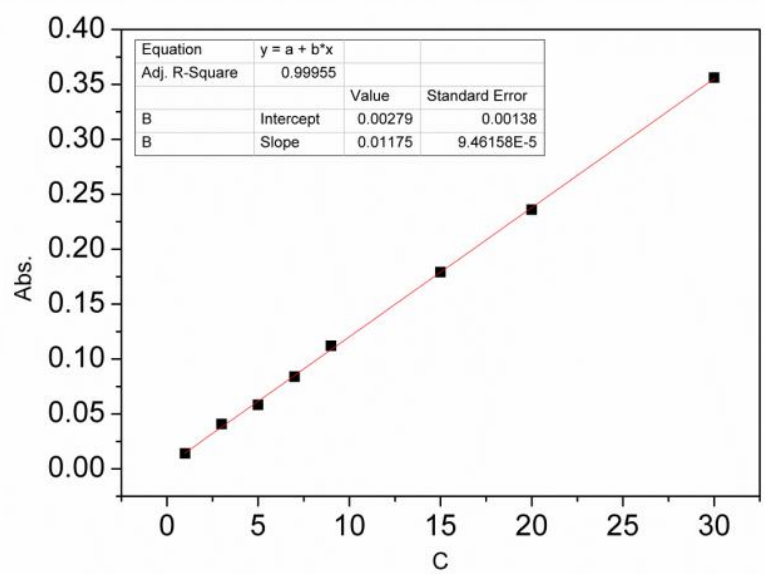

Figure S28. The relationship of concentration and absorbance of congo red.

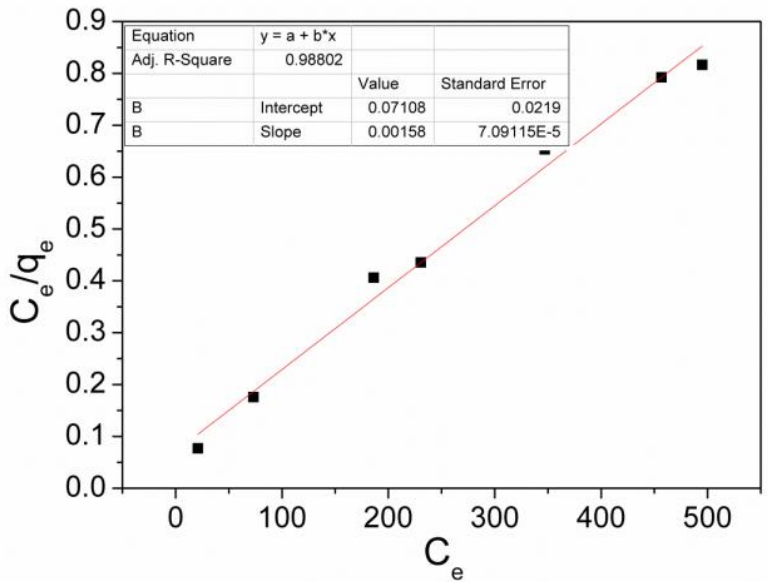

Figure S29. The Langmuir isotherm of Poly(BVIm-DVB-Cl) on congo red.

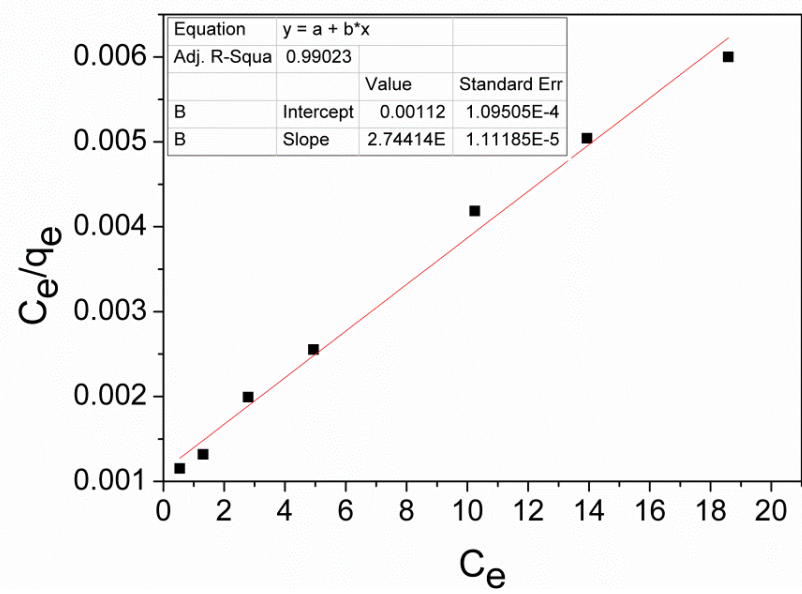

Figure S30. The Langmuir isotherm of Poly(BVIm-DVB- $\left.\mathrm{BPhF}_{3}\right)-\mathrm{Cl}$ on congo red. 


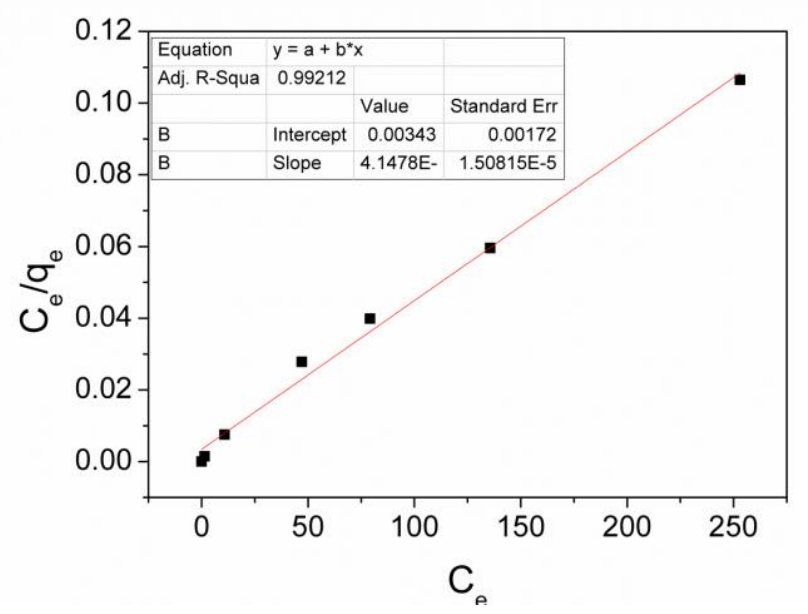

Figure S31. The Langmuir isotherm of Poly(BVIm-DVB-BPh 4$)-C l$ on congo red.

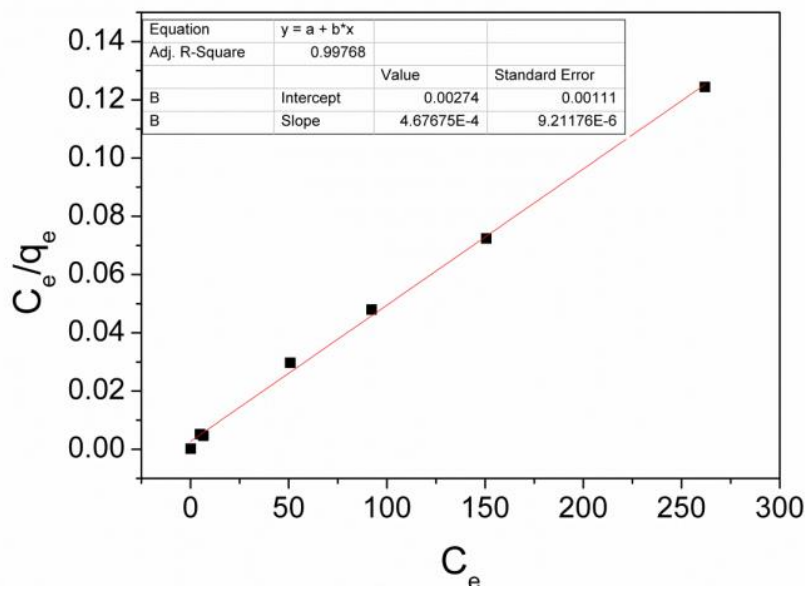

Figure S32. The Langmuir isotherm of Poly(BVIm-DVB-BNap 4 )-Cl on congo red.

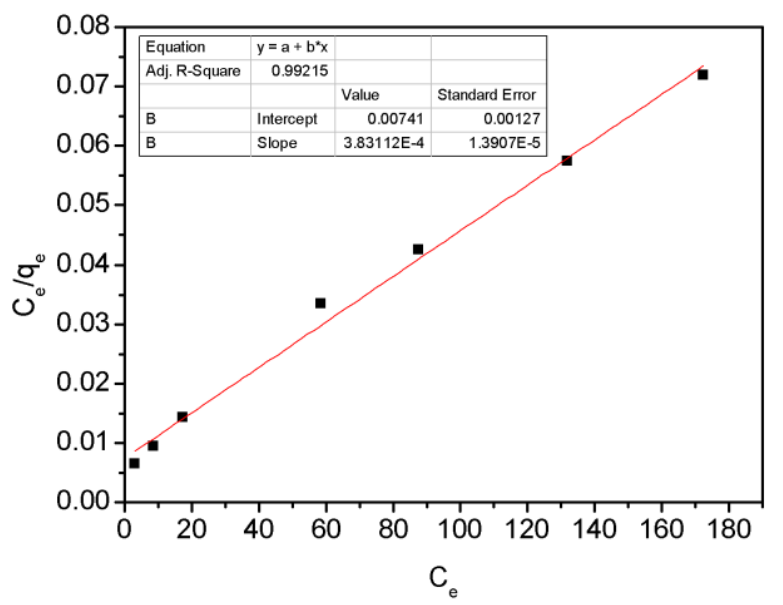

Figure S33. The Langmuir isotherm of Poly(BVIm-DVB-BPhF 3 -OAc on congo red. 


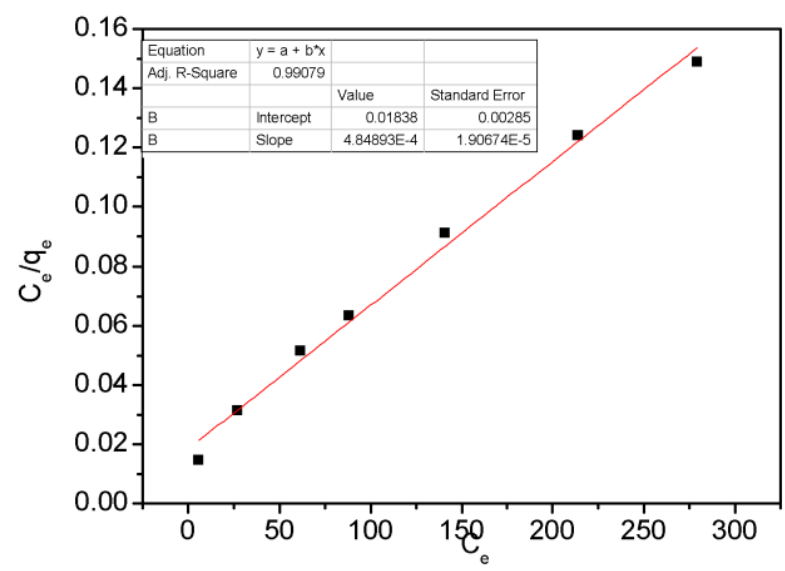

Figure S34. The Langmuir isotherm of Poly(BVIm-DVB-BPh 4$)-O A c$ on congo red.

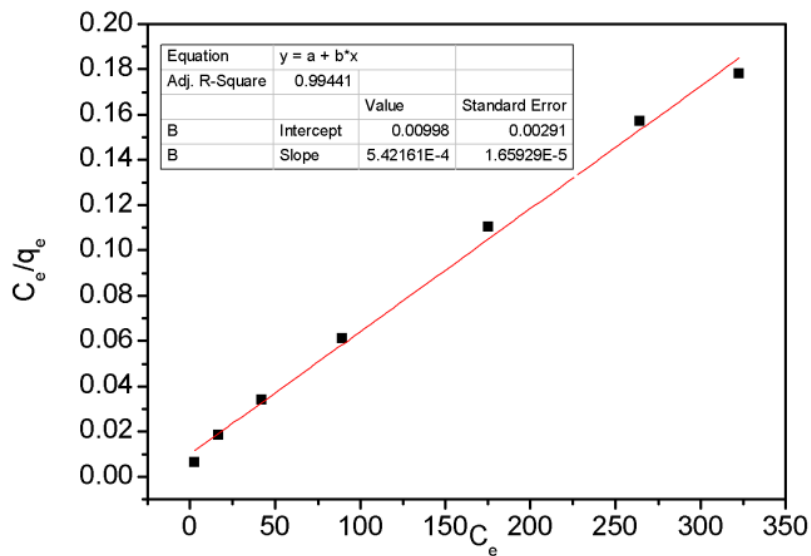

Figure S35. The Langmuir isotherm of Poly(BVIm-DVB-BNap)-OAc on congo red.

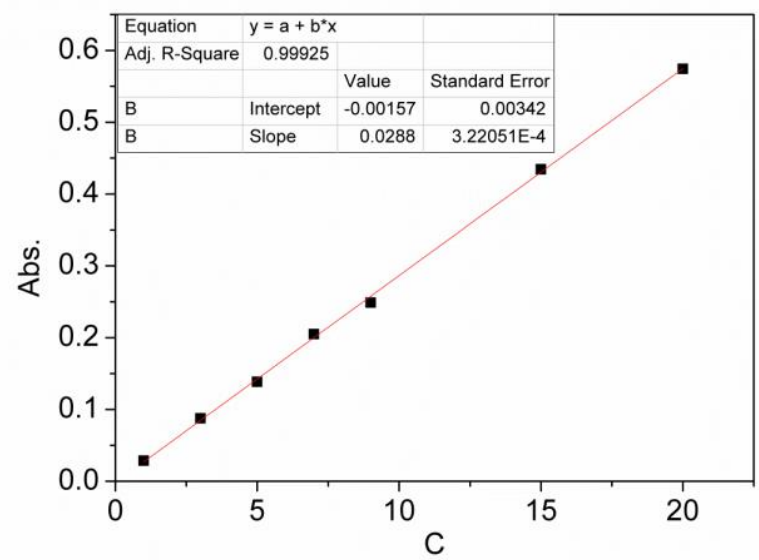

Figure S36. The relationship of concentration and absorbance of methyl blue. 


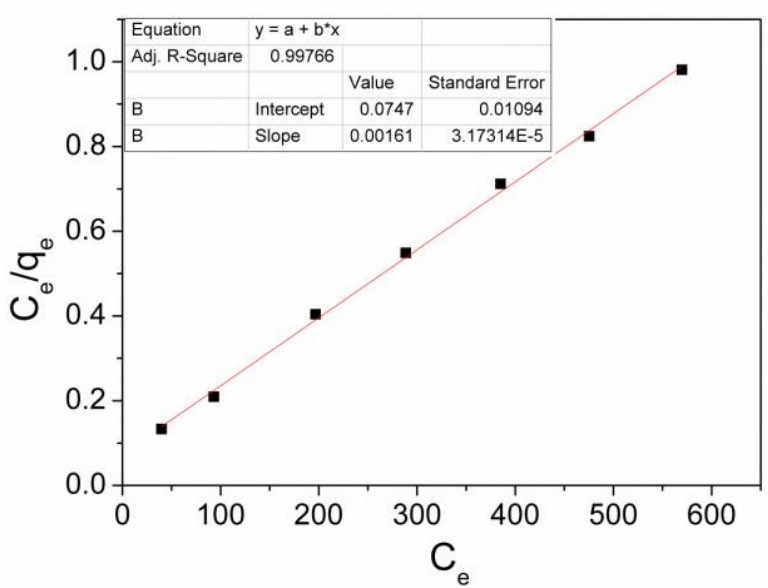

Figure S37. The Langmuir isotherm of Poly(BVIm-DVB-Cl) on methyl blue.

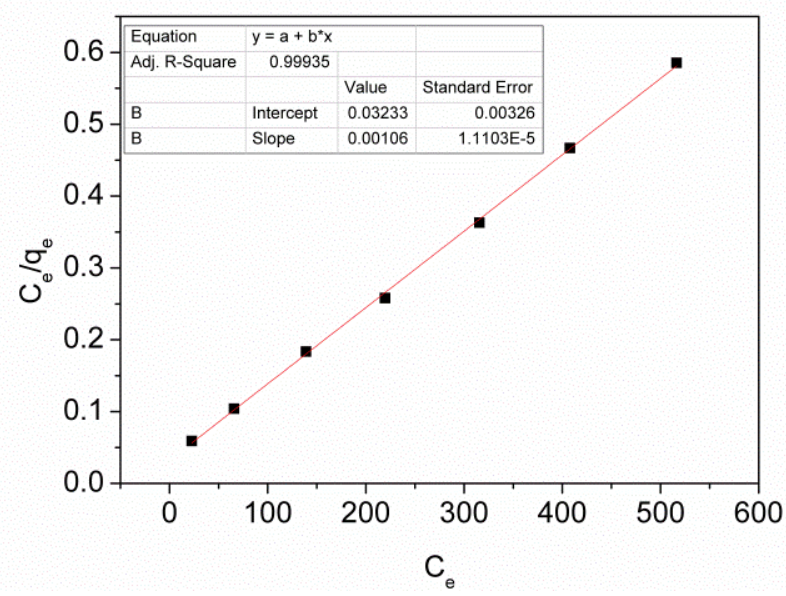

Figure S38. The Langmuir isotherm of Poly(BVIm-DVB-BPhF 3$)-\mathrm{Cl}$ on methyl blue.

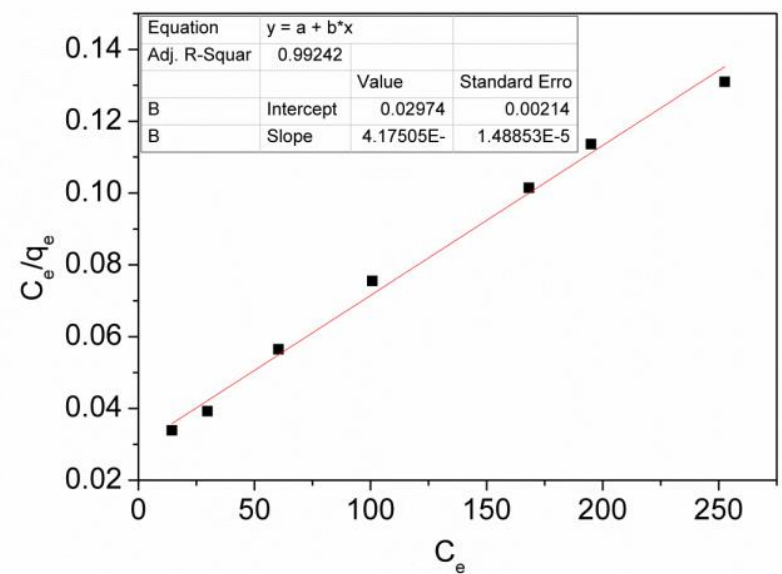

Figure S39. The Langmuir isotherm of Poly(BVIm-DVB-BPh 4 )-Cl on methyl blue. 


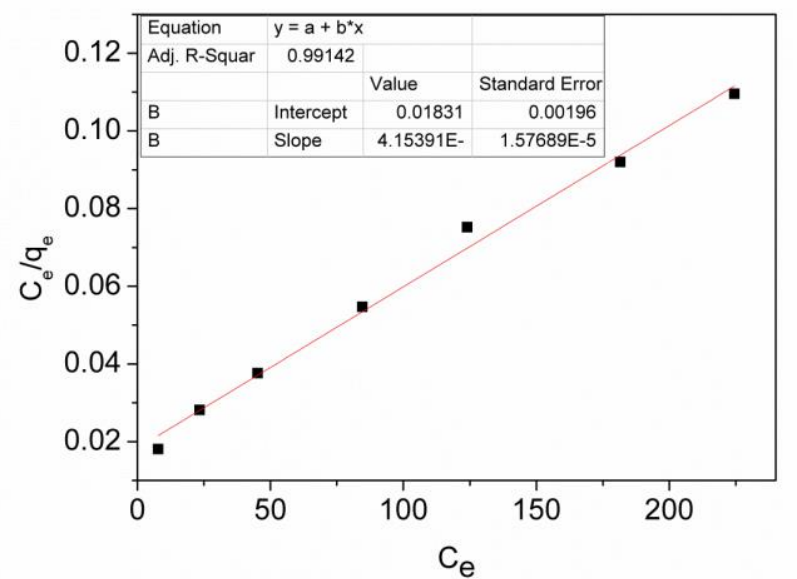

Figure S40. The Langmuir isotherm of Poly(BVIm-DVB-BNap4)-Cl on methyl blue.

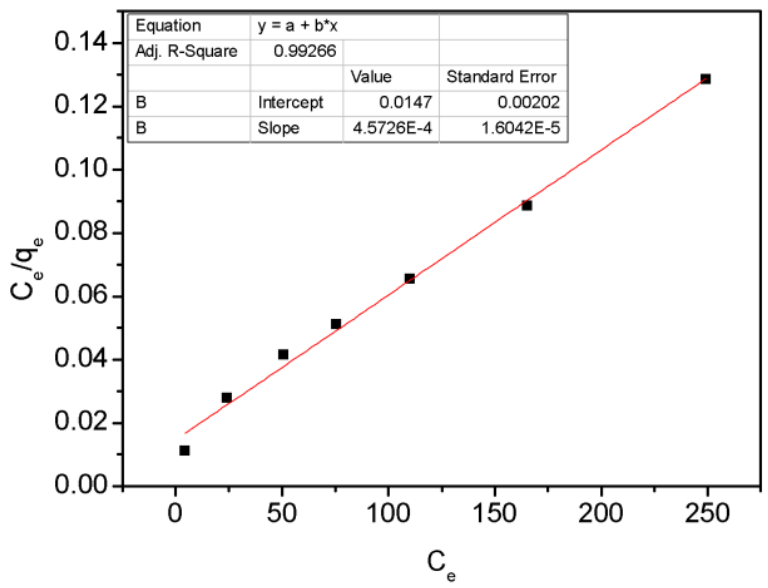

Figure S41. The Langmuir isotherm of Poly(BVIm-DVB-BPh 4$)-O A c$ on methyl blue

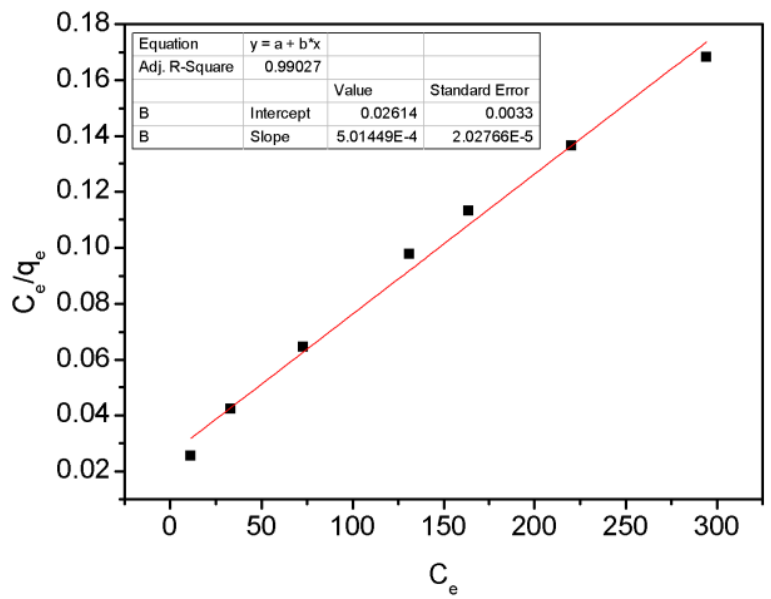

Figure S42. The Langmuir isotherm of Poly(BVIm-DVB-BNap4)-OAc on methyl blue. 


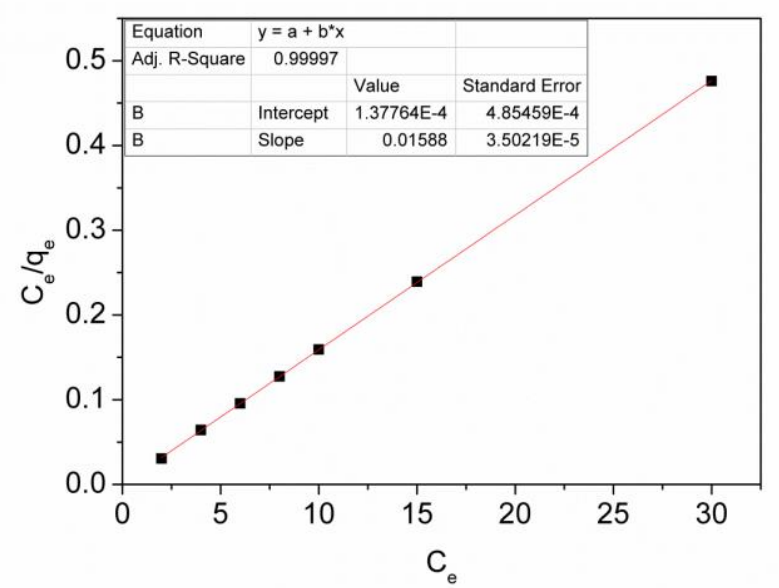

Figure S43. The relationship of concentration and absorbance of sirius red.

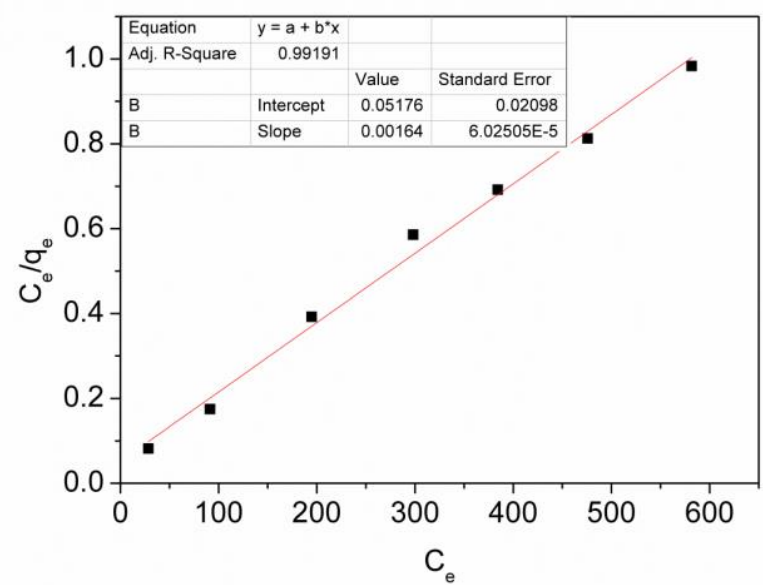

Figure S44. The Langmuir isotherm of Poly(BVIm-DVB-Cl) on sirius red.

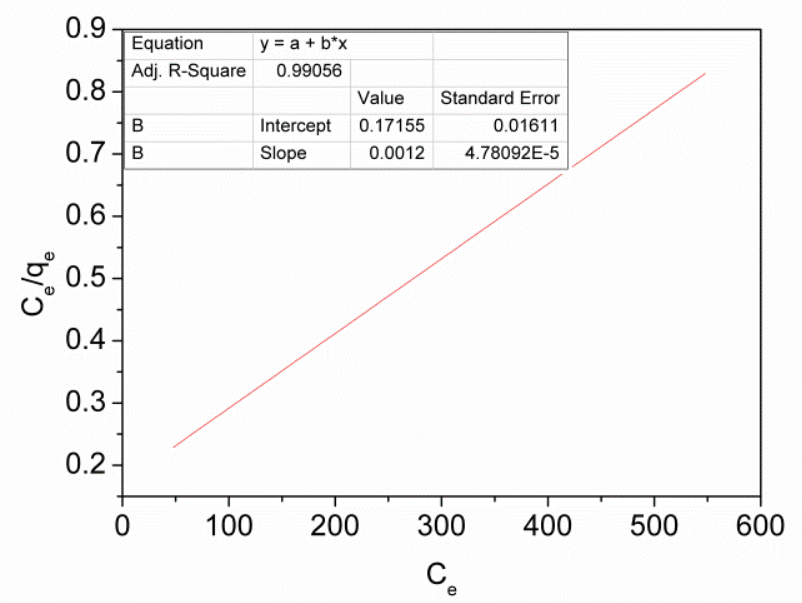

Figure S45. The Langmuir isotherm of Poly(BVIm-DVB-BPhF $)-C l$ on sirius red. 


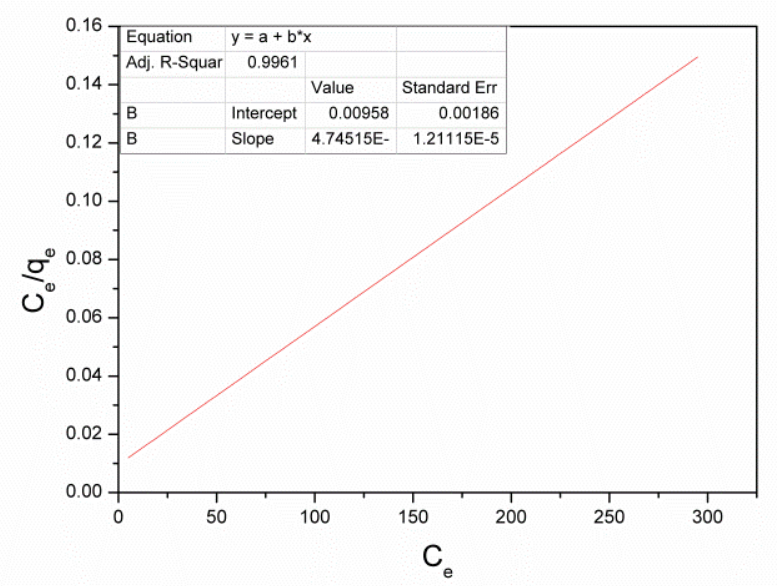

Figure S46. The Langmuir isotherm of Poly(BVIm-DVB-BPh 4 )-Cl on sirius red.

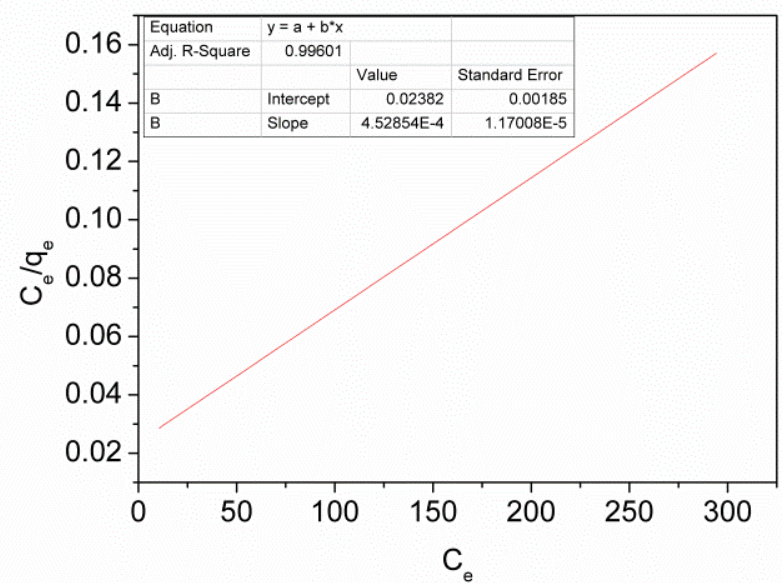

Figure S47. The Langmuir isotherm of Poly(BVIm-DVB-BNap4)-Cl on sirius red.

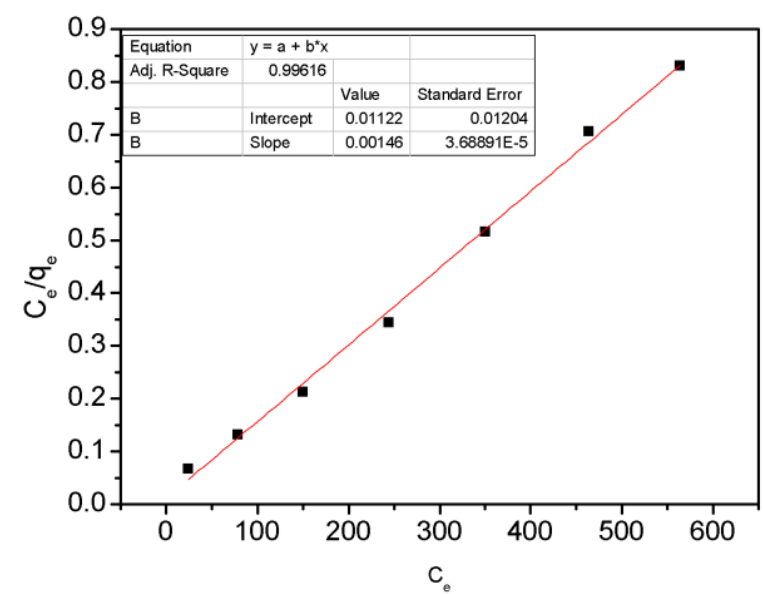

Figure S48. The Langmuir isotherm of Poly(BVIm-DVB-BPhF 3 -OAc on sirius red. 


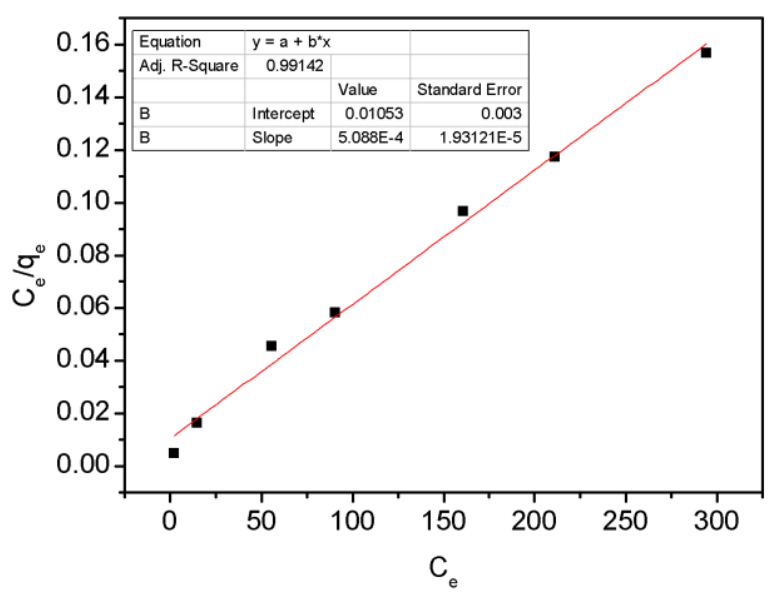

Figure S49. The Langmuir isotherm of Poly(BVIm-DVB-BPh 4$)-O A c$ on sirius red

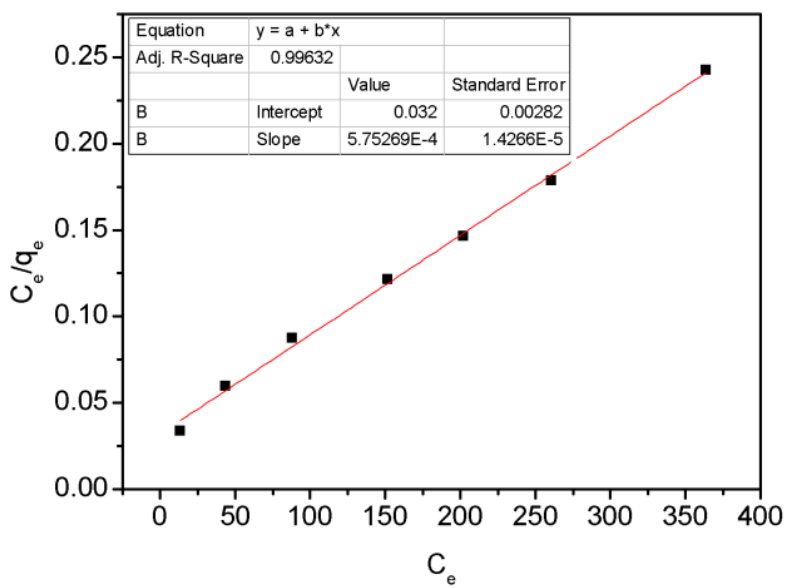

Figure S50. The Langmuir isotherm of Poly(BVIm-DVB-BNap4)-OAc on sirius red

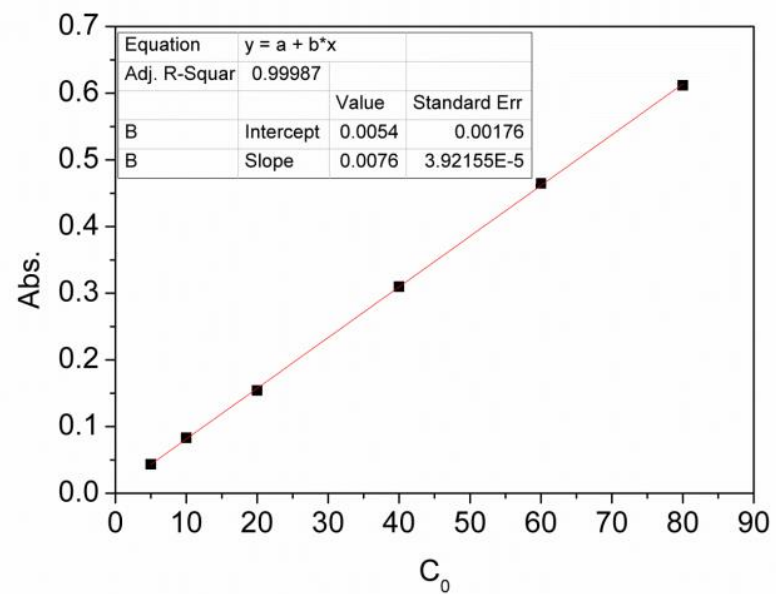

Figure S51. The relationship of concentration and absorbance of sodium lignosulphonate. 


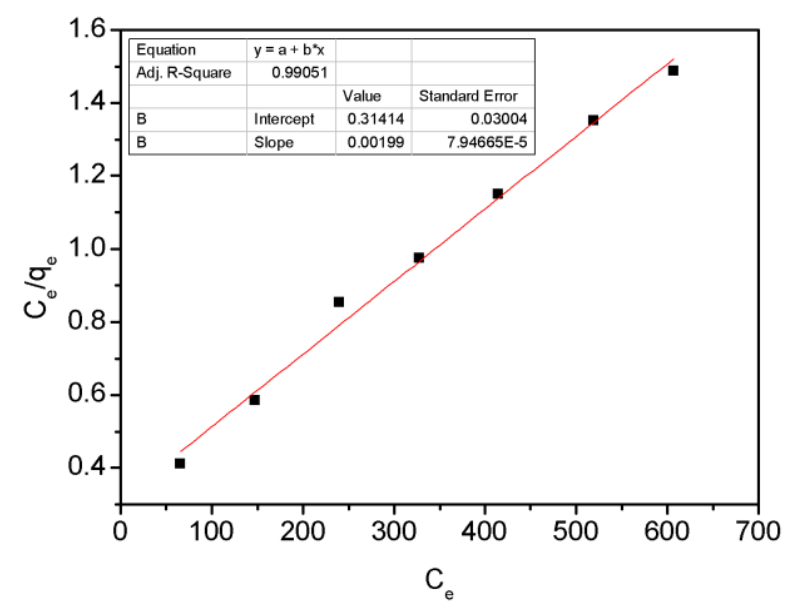

Figure S52. The Langmuir isotherm of Poly(BVIm-DVB-Cl) on sodium lignosulphonate.

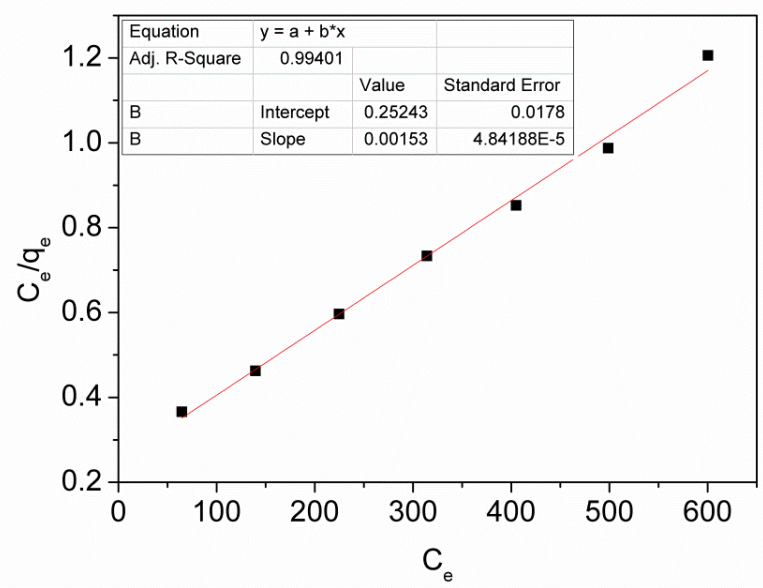

Figure S53. The Langmuir isotherm of Poly(BVIm-DVB- $\left.\mathrm{BPhF}_{3}\right)-\mathrm{Cl}$ on sodium lignosulphonate.

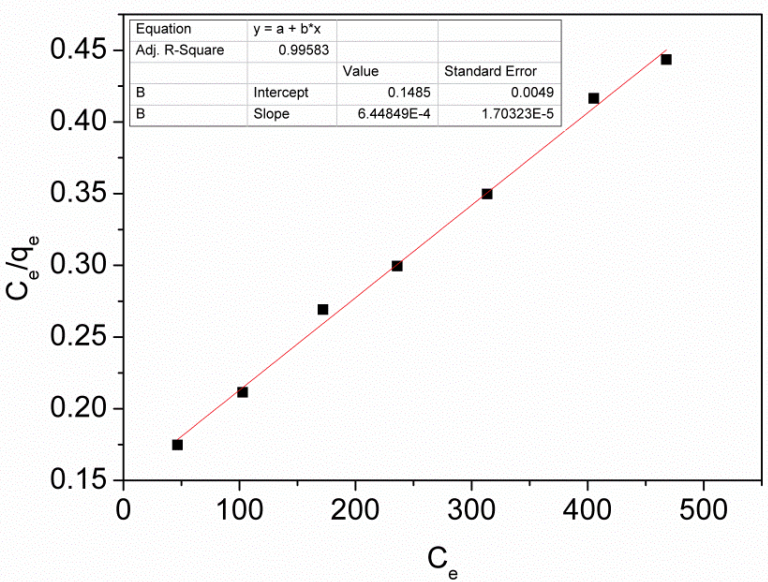

Figure S54. The Langmuir isotherm of Poly(BVIm-DVB-BPh 4 )-Cl on sodium lignosulphonate. 


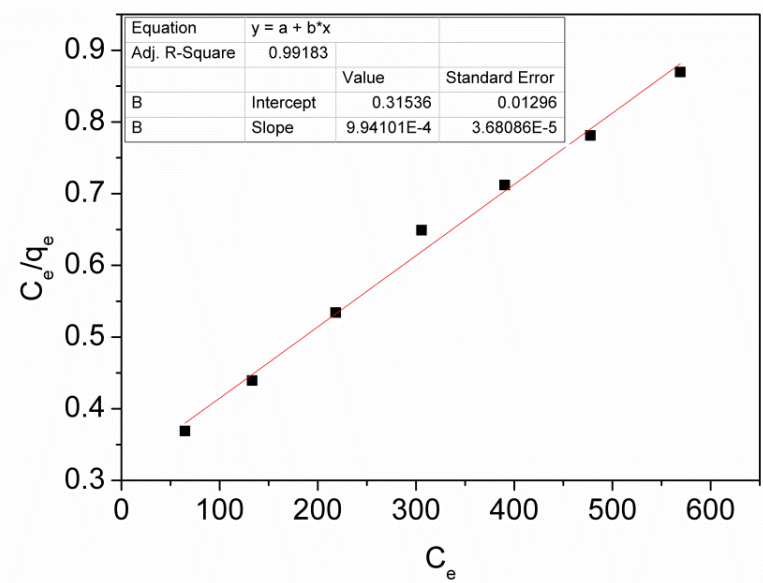

Figure S55. The Langmuir isotherm of Poly(BVIm-DVB-BPh 4$)-C l$ on sodium lignosulphonate.

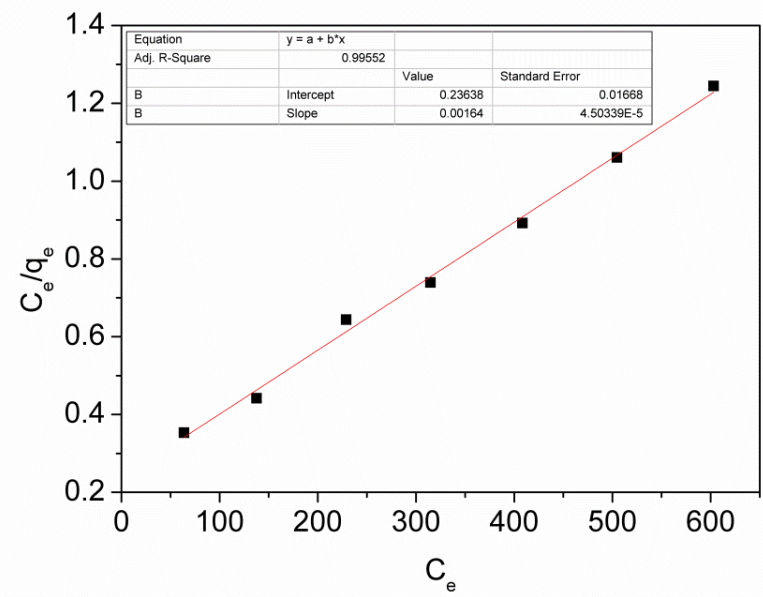

Figure S56. The Langmuir isotherm of Poly(BVIm-DVB-BPhF 3 )-OAc on sodium lignosulphonate.

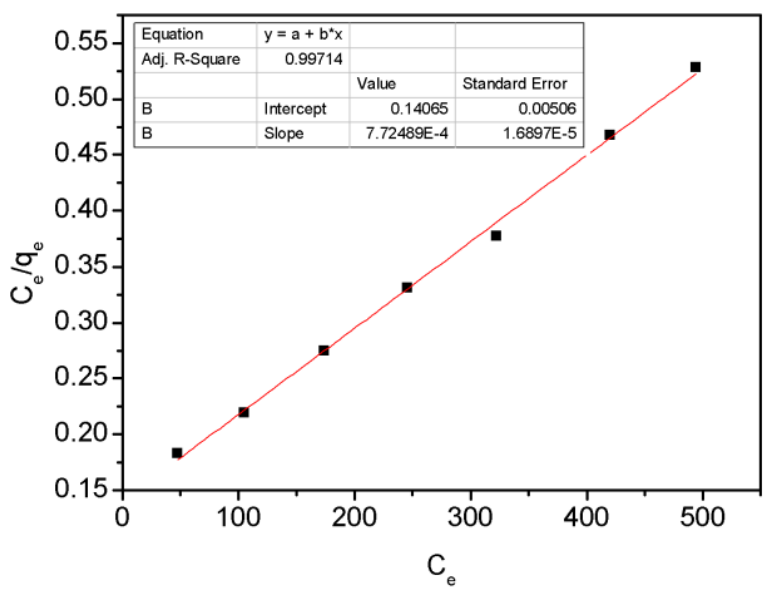

Figure S57. The Langmuir isotherm of Poly(BVIm-DVB-BPh 4 )-OAc on sodium lignosulphonate. 


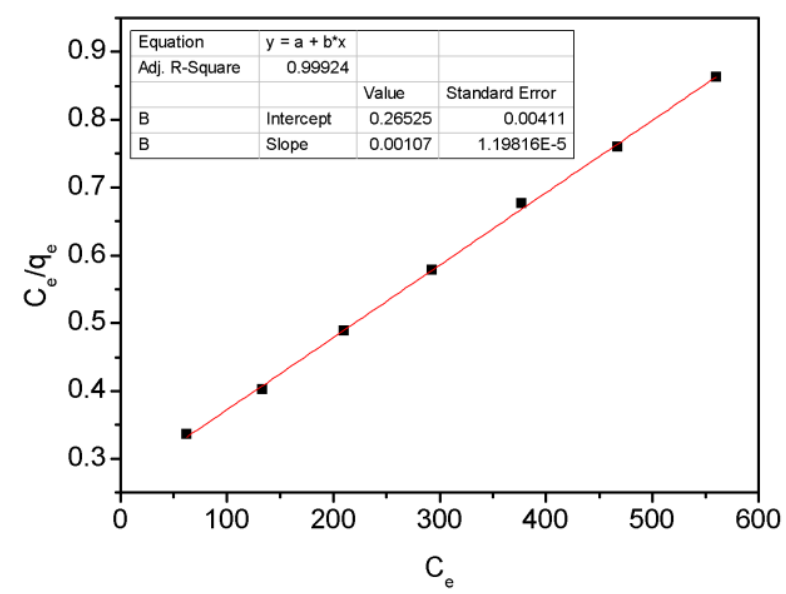

Figure S58. The Langmuir isotherm of Poly(BVIm-DVB-BNap 4 )-OAc on sodium lignosulphonate.

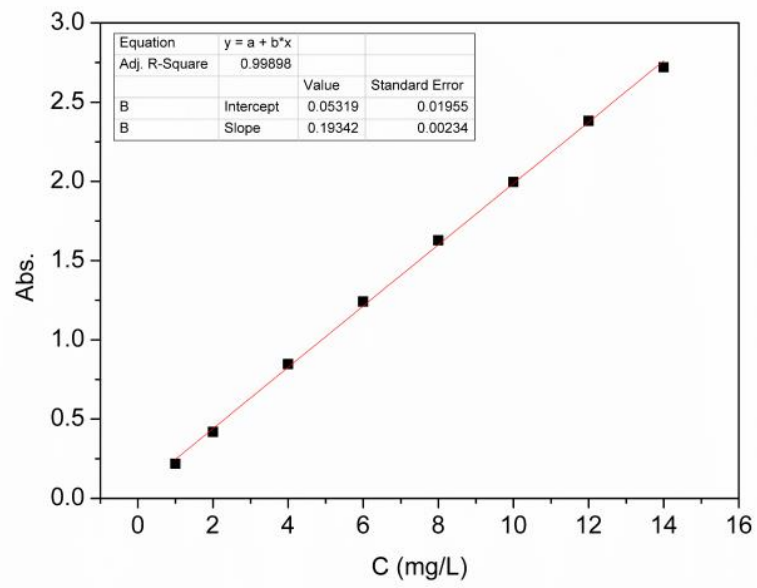

Figure S59. The relationship of concentration and absorbance of rhodamine B.

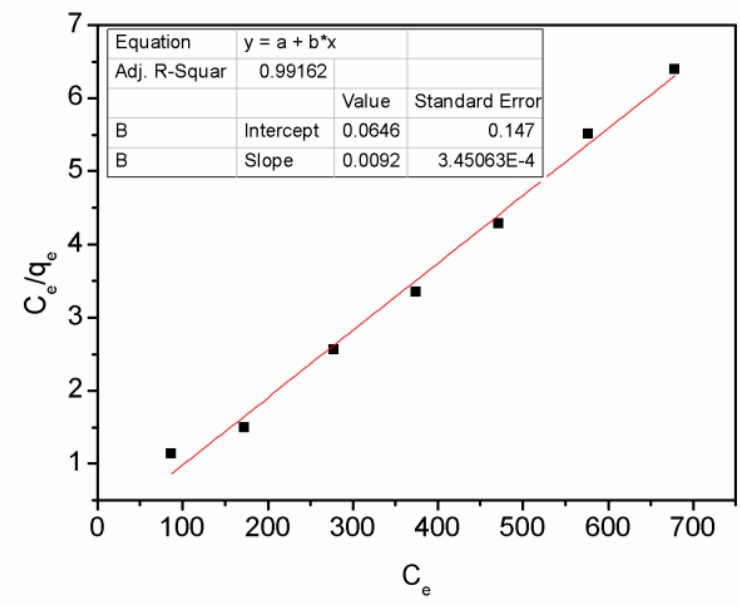

Figure S60. The Langmuir isotherm of Poly(BVIm-DVB-Cl) on rhodamine B. 


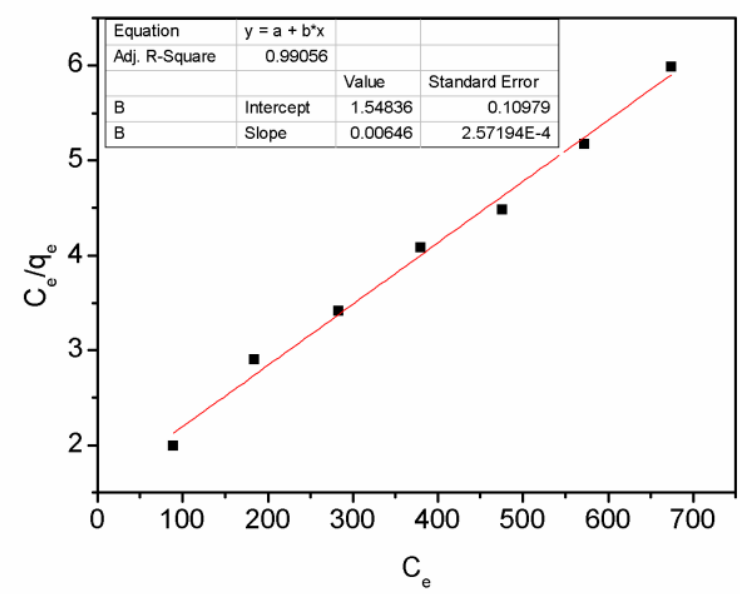

Figure S61. The Langmuir isotherm of Poly(BVIm-DVB-BPhF 3 -Cl on rhodamine B.

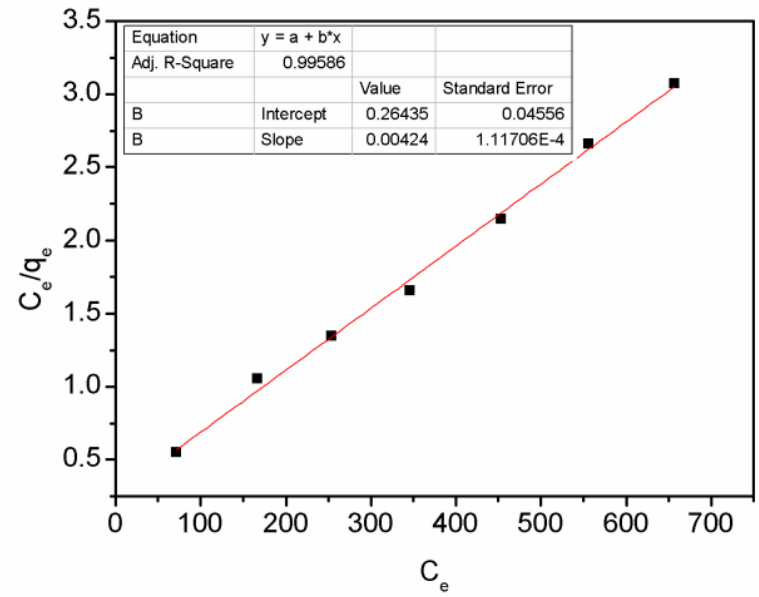

Figure S62. The Langmuir isotherm of Poly(BVIm-DVB- $\left.\mathrm{BPh}_{4}\right)-\mathrm{Cl}$ on rhodamine B.

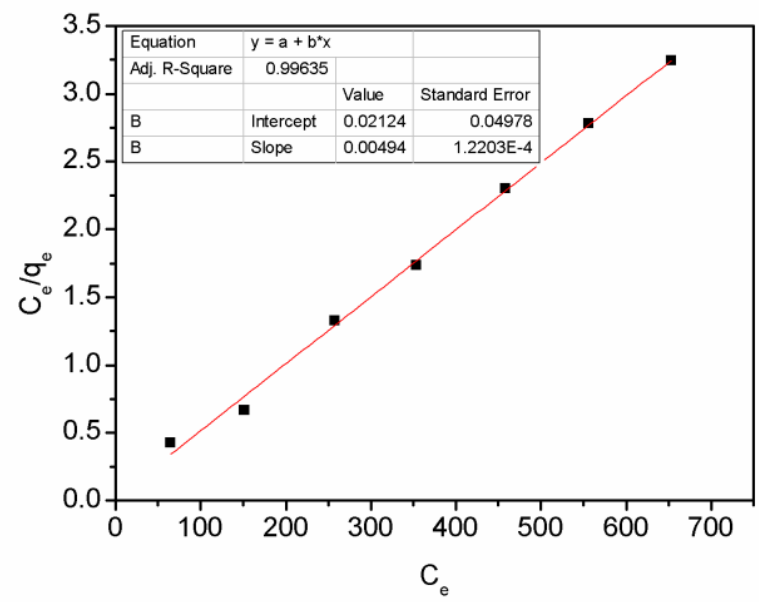

Figure S63. The Langmuir isotherm of Poly(BVIm-DVB-BNap 4 )-Cl on rhodamine B. 


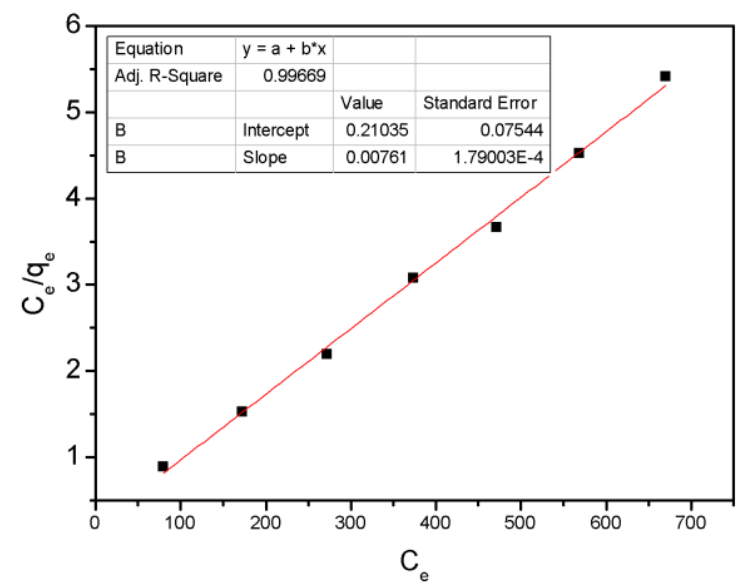

Figure S64. The Langmuir isotherm of Poly(BVIm-DVB-BPhF 3 )-OAc on rhodamine $\mathrm{B}$.

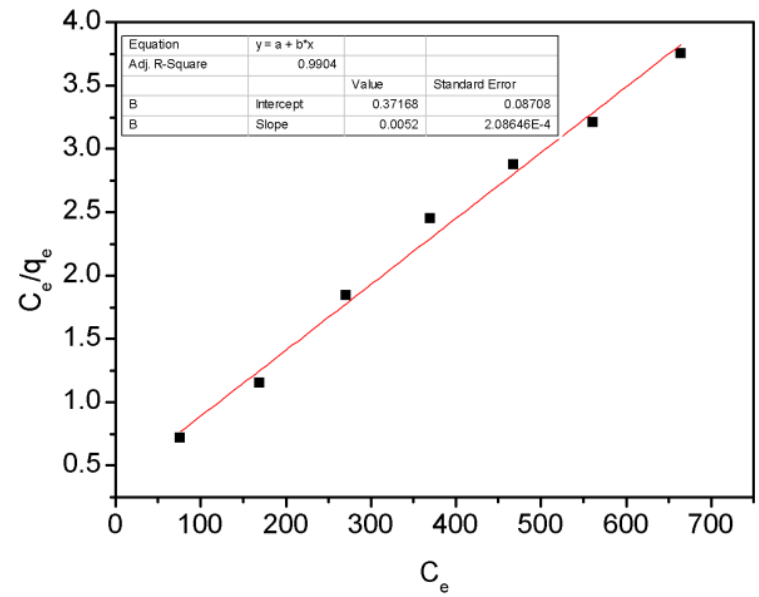

Figure S65. The Langmuir isotherm of Poly(BVIm-DVB-BPh 4 )-OAc on rhodamine B.

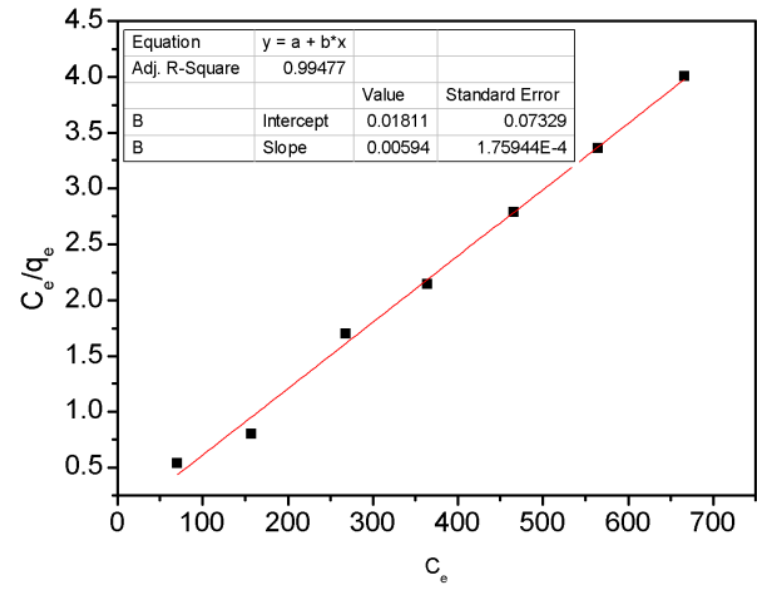

Figure S66. The Langmuir isotherm of Poly(BVIm-DVB-BNap 4 )-OAc on rhodamine B. 


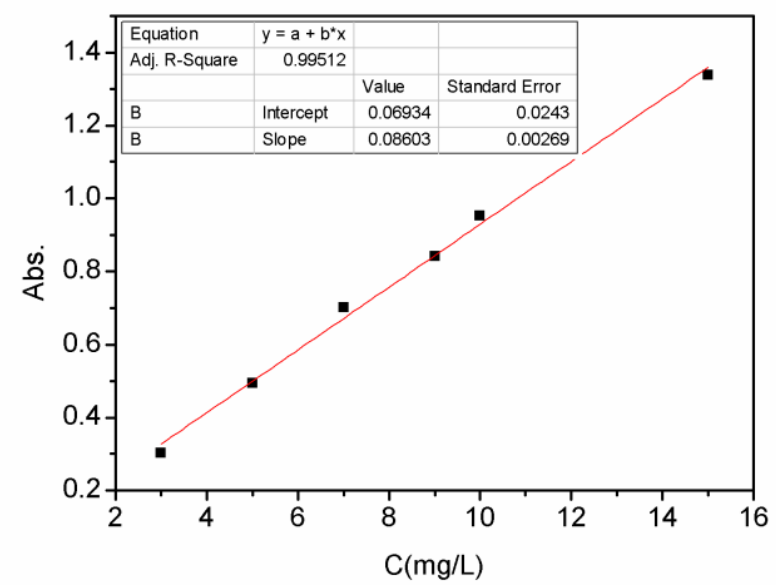

Figure S67. The relationship of concentration and absorbance of indigo.

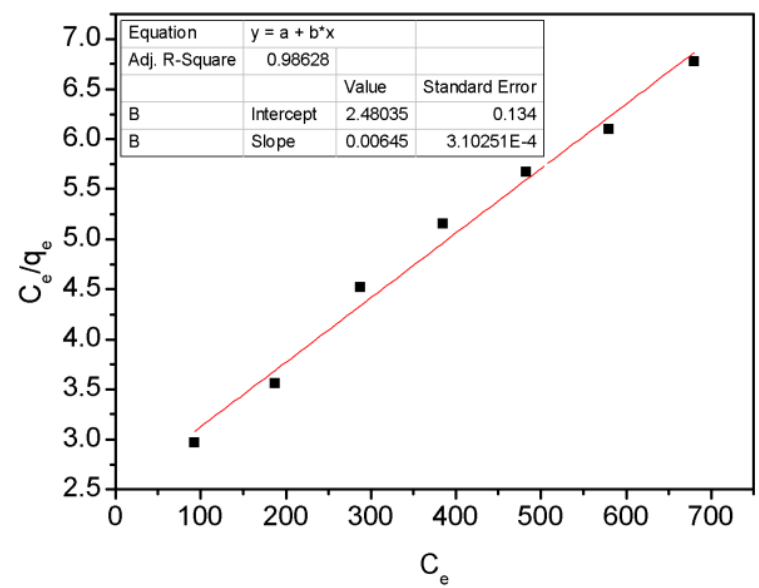

Figure S68. The Langmuir isotherm of Poly(BVIm-DVB-Cl) on indigo.

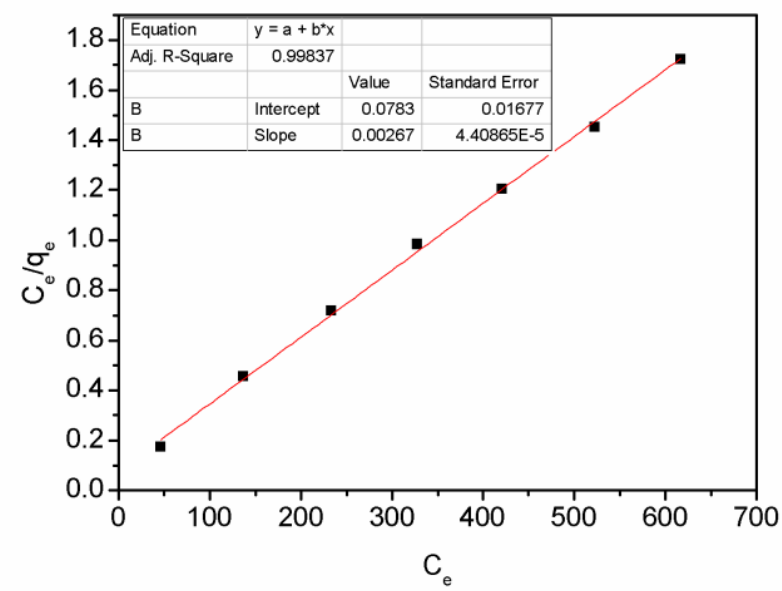

Figure S69. The Langmuir isotherm of Poly(BVIm-DVB-BPhF 3 )-Cl on indigo. 


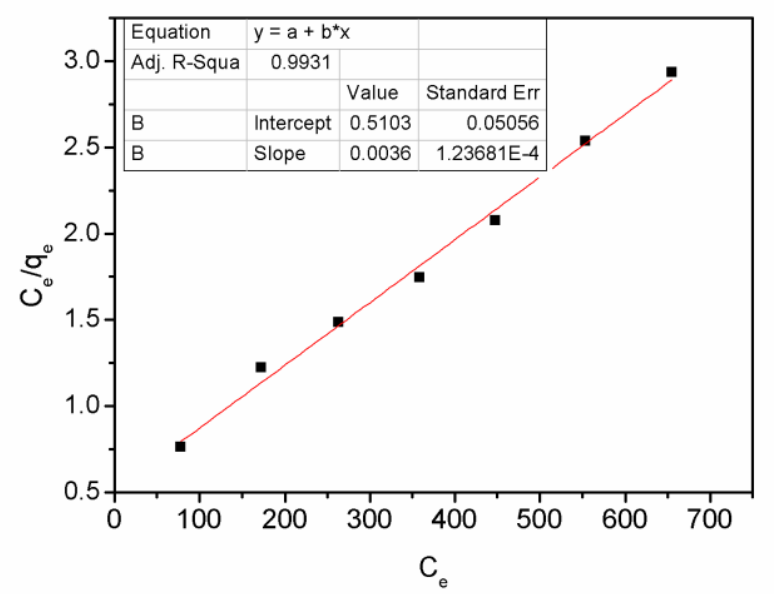

Figure S70. The Langmuir isotherm of Poly(BVIm-DVB- $\left.\mathrm{BPh}_{4}\right)-\mathrm{Cl}$ on indigo.

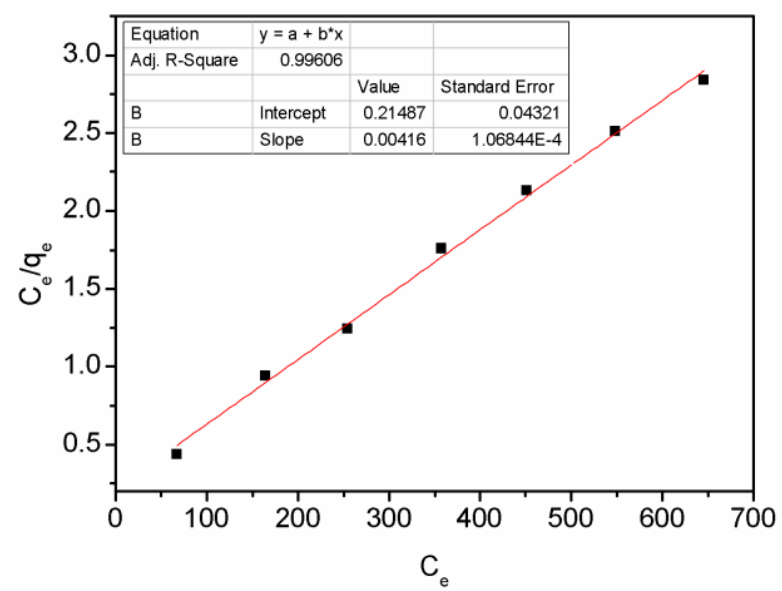

Figure S71. The Langmuir isotherm of Poly(BVIm-DVB-BNap4)-Cl on indigo.

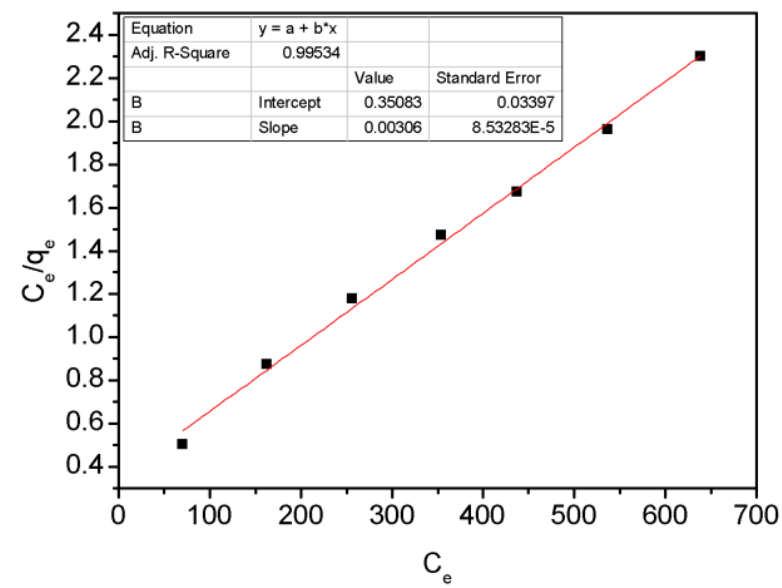

Figure S72. The Langmuir isotherm of Poly(BVIm-DVB-BPhF 3 -OAc on indigo. 


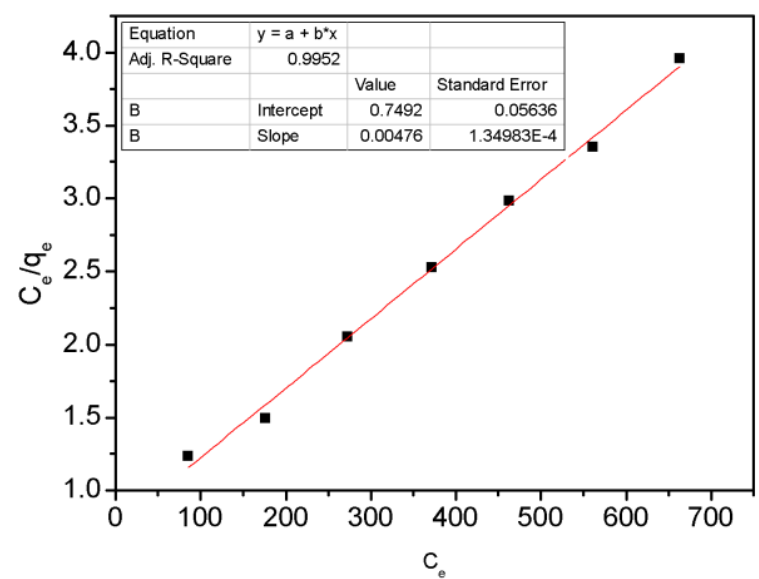

Figure S73. The Langmuir isotherm of Poly(BVIm-DVB-BPh 4$)-O A c$ on indigo.

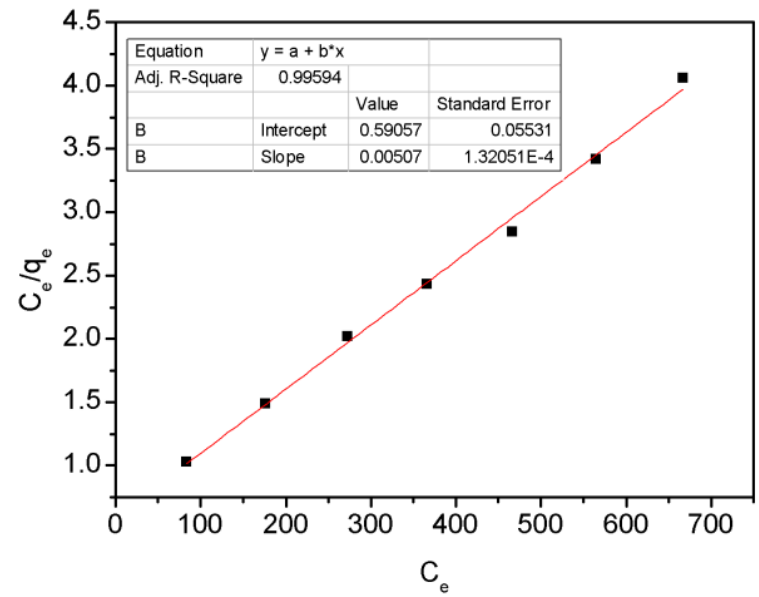

Figure S74. The Langmuir isotherm of Poly(BVIm-DVB-BNap4)-OAc on indigo. 